TRANSACTIONS OF THE

AMERICAN MATHEMATICAL SOCIETY

Volume 356, Number 11, Pages 4655-4685

S 0002-9947(04)03502-0

Article electronically published on May 10, 2004

\title{
CONVOLUTION ROOTS OF RADIAL POSITIVE DEFINITE FUNCTIONS WITH COMPACT SUPPORT
}

\author{
WERNER EHM, TILMANN GNEITING, AND DONALD RICHARDS
}

\begin{abstract}
A classical theorem of Boas, Kac, and Krein states that a characteristic function $\varphi$ with $\varphi(x)=0$ for $|x| \geq \tau$ admits a representation of the form

$$
\varphi(x)=\int u(y) \overline{u(y+x)} \mathrm{d} y, \quad x \in \mathbb{R},
$$
\end{abstract}

where the convolution root $u \in L^{2}(\mathbb{R})$ is complex-valued with $u(x)=0$ for $|x| \geq \tau / 2$. The result can be expressed equivalently as a factorization theorem for entire functions of finite exponential type. This paper examines the BoasKac representation under additional constraints: If $\varphi$ is real-valued and even, can the convolution root $u$ be chosen as a real-valued and/or even function? A complete answer in terms of the zeros of the Fourier transform of $\varphi$ is obtained. Furthermore, the analogous problem for radially symmetric functions defined on $\mathbb{R}^{d}$ is solved. Perhaps surprisingly, there are compactly supported, radial positive definite functions that do not admit a convolution root with half-support. However, under the additional assumption of nonnegativity, radially symmetric convolution roots with half-support exist. Further results in this paper include a characterization of extreme points, pointwise and integral bounds (Turán's problem), and a unified solution to a minimization problem for compactly supported positive definite functions. Specifically, if $f$ is a probability density on $\mathbb{R}^{d}$ whose characteristic function $\varphi$ vanishes outside the unit ball, then

$$
\int|x|^{2} f(x) \mathrm{d} x=-\Delta \varphi(0) \geq 4 j_{(d-2) / 2}^{2}
$$

where $j_{\nu}$ denotes the first positive zero of the Bessel function $J_{\nu}$, and the estimate is sharp. Applications to spatial moving average processes, geostatistical simulation, crystallography, optics, and phase retrieval are noted. In particular, a real-valued half-support convolution root of the spherical correlation function in $\mathbb{R}^{2}$ does not exist.

\section{INTRODUCTION}

1.1. Convolution roots of characteristic functions. A probability density $f$, being nonnegative, can always be written as a square. Clearly then, its characteristic function or Fourier transform $\varphi$ admits a convolution type representation, a result that is known as the Wiener-Khintchine criterion [22, p. 640]. Matters become more subtle if support conditions are added. The key result here is a theorem of Boas and Kac 9], stating that a characteristic function with compact support always has a half-support convolution root: Suppose the characteristic function $\varphi$ is such that $\varphi(x)=0$ for $|x| \geq \tau$. Then there exists a complex-valued square-integrable

Received by the editors April 10, 2003 and, in revised form, September 2, 2003.

2000 Mathematics Subject Classification. Primary 42A38, 42A82, 60E10. 
function $u$ with $u(x)=0$ for $|x| \geq \tau / 2$, and such that

$$
\varphi=u \tilde{*} u
$$

where

$$
u \widetilde{*} u(x)=\int u(y) \overline{u(y+x)} \mathrm{d} y
$$

denotes the folded-over (self-)convolution of $u$. We call any function $u$ satisfying these conditions a Boas-Kac root of $\varphi$.

If $\varphi$ is known to have additional, special properties, one might ask which additional properties this entails for the Boas-Kac roots $u$. In particular, if $\varphi$ is an even function, can $u$ be selected so as to be even, too? Can $u$ be chosen as real-valued, or as both real-valued and even? These questions are motivated mainly by their relevance to the higher-dimensional case of radial positive definite functions. Under what conditions do such functions admit Boas-Kac (half-support) convolution roots with useful additional properties? This paper provides a complete solution to the existence problem for even (radial) and/or real-valued convolution roots. The results have applications in various areas and provide interesting examples and counterexamples.

1.2. Outline and results. We write $x \cdot y=\sum_{l=1}^{d} x_{l} y_{l}$ for the inner product of $x=\left(x_{1}, \ldots, x_{d}\right)$ and $y=\left(y_{1}, \ldots, y_{d}\right)$ in $\mathbb{R}^{d}$, and we use operator notation for the Fourier transform of a complex-valued function $g$ on $\mathbb{R}^{d}$,

$$
\mathcal{F}_{d} g(y)=\int e^{i y \cdot x} g(x) \mathrm{d} x, \quad \mathcal{F}_{d}^{-1} g(x)=\frac{1}{(2 \pi)^{d}} \int e^{-i x \cdot y} g(y) \mathrm{d} y .
$$

If $d=1$, we frequently put $\mathcal{F}_{1}=\mathcal{F}$ and $\mathcal{F}_{1}^{-1}=\mathcal{F}^{-1}$. Note that $g$ is a real-valued function if and only if $h=\mathcal{F}_{d} g$ is Hermitian, i.e., $h(-x)=\overline{h(x)}$ for $x \in \mathbb{R}^{d}$. If $f$ is a probability density, we denote its characteristic function or Fourier transform by $\varphi=\mathcal{F}_{d} f$. Then $\varphi$ is continuous with $\varphi(0)=1$ as well as positive definite, the latter meaning that

$$
\sum_{k=1}^{n} \sum_{k=l}^{n} c_{k} \overline{c_{l}} \varphi\left(x_{k}-x_{l}\right) \geq 0
$$

for all finite systems of complex numbers $c_{1}, \ldots, c_{n}$ and points $x_{1}, \ldots, x_{n} \in \mathbb{R}^{d}$. Conversely, Bochner's theorem [10] states that every such function $\varphi$ is the Fourier transform of a probability measure $F$ on $\mathbb{R}^{d}$. If $\varphi$ is integrable, then $F$ has a continuous density given by $f=\mathcal{F}_{d}^{-1} \varphi$. Since a positive definite function is Hermitian, the following three conditions are equivalent for densities $f: f$ is symmetric; $\varphi$ is symmetric; and $\varphi$ is real-valued.

Let $\varphi$ be a characteristic function on $\mathbb{R}$ with $\varphi(x)=0$ for $|x| \geq \tau$. Its probability density $f=\mathcal{F}^{-1} \varphi$ admits an analytic continuation to the entire function given by

$$
f(z)=\frac{1}{2 \pi} \int_{\mathbb{R}} e^{-i z x} \varphi(x) \mathrm{d} x, \quad z \in \mathbb{C} .
$$

A straightforward estimate shows that $f$ satisfies the growth condition

$$
\limsup _{r \rightarrow \infty} r^{-1} \log \max _{|z|=r}|f(z)| \leq \tau
$$

so that $f$ is an entire function of finite exponential type $\tau$. This is a consequence of the Paley-Wiener theorem [52, p. 13] which characterizes the class of entire 
TABLe 1. Existence and Properties of Boas-Kac Roots: Summary of Results in Theorem $2.1(d=1, \varphi$ even $)$ and Theorem $3.1(d \geq 2$, $\varphi$ radial).

\begin{tabular}{|l||l|l|l|}
\hline Dimension & \multicolumn{1}{|c|}{$d=1$} & $d=2$ & \multicolumn{1}{c|}{$d \geq 3$} \\
\hline \hline Existence & & \multicolumn{1}{|c}{$(\mathrm{B})$} & $(\mathrm{A})(\mathrm{B})$ \\
\hline Real-Valued & & $(\mathrm{A})(\mathrm{B})(\mathrm{C})$ & $(\mathrm{A})(\mathrm{B})(\mathrm{C})$ \\
\hline Radial & (A) $(\mathrm{B})$ & $(\mathrm{A})(\mathrm{B})$ & $(\mathrm{A})(\mathrm{B})$ \\
\hline Real-Valued and Radial & (A) $(\mathrm{B})(\mathrm{C})$ & $(\mathrm{A})(\mathrm{B})(\mathrm{C})$ & $(\mathrm{A})(\mathrm{B})(\mathrm{C})$ \\
\hline
\end{tabular}

functions of finite exponential type $\tau$ that are square integrable on $\mathbb{R}$ : A function $g(z), z \in \mathbb{C}$, belongs to this class if and only if it can be represented as

$$
g(z)=\frac{1}{2 \pi} \int_{\mathbb{R}} e^{-i z x} \gamma(x) \mathrm{d} x
$$

for some $\gamma \in L^{2}(\mathbb{R})$ with $\gamma(x)=0$ for $|x| \geq \tau$. Hence, the Boas-Kac convolution theorem can be stated equivalently as a factorization theorem for entire functions of finite exponential type: If $f$ is an entire function of finite exponential type $\tau$ that is nonnegative and integrable on $\mathbb{R}$, then there exists an entire function $\psi$ of exponential type $\tau / 2$ such that

$$
f(z)=\psi(z) \overline{\psi(\bar{z})}, \quad z \in \mathbb{C} .
$$

The connection between $f$ and $\varphi$, and between $\psi$ and $u$, respectively, is furnished by the Fourier transform, in terms of which the theorem assumes a third equivalent form: If $f$ is a band-limited probability density with spectrum contained in $[-\tau, \tau]$, then there exists a function $\psi \in L^{2}(\mathbb{R})$ with spectrum contained in $[-\tau / 2, \tau / 2]$ such that $f=|\psi|^{2}$ on $\mathbb{R}$.

Here we focus on conditions under which the Boas-Kac root $u$ of $\varphi$ can be chosen as a real-valued and/or even function. The basic conditions required for our results involve the zeros of the analytic continuation of the probability density $f$ associated with $\varphi$.

Condition (A). If $f$ has a zero at the origin, then its order is a multiple of 4 .

Condition (B). Any purely imaginary zero of $f$ has even order.

Condition (C). Any zero of $f$ that is neither real nor purely imaginary has even order.

The results are stated in Theorem 2.1] and the respective necessary and sufficient conditions are summarized in the left-hand column of Table 1.

In Section 3 we consider the analogous problem in dimensions $d \geq 2$, for radially symmetric positive definite functions with compact support. The corresponding density $f$ then is radial, too, and the relevant conditions can be stated in terms of the zeros of the analytic continuation of the radial part of $f$. These results are detailed in Theorem 3.1, and summarized in the second and third columns of Table 1 Unlike in the case $d=1$ and contrary to previous claims [59], the existence of a Boas-Kac root is not guaranteed without further restrictions if $d \geq 2$, and explicit counterexamples will be given. However, a Boas-Kac root does exist whenever $\varphi$ is nonnegative. These results and the examples presented later on suggest that existence of a real-valued Boas-Kac root is an exception rather than the rule if $d \geq 2$. 
Section 4 turns to pointwise and integral bounds for positive definite functions with compact support. Under the heading of Turán's problem, questions of this type have recently been studied by Arestov, Berdysheva, and Berens [4, 5] 6] and Kolountzakis and Révész [41, 42], among others. Theorem 4.1 presents pointwise bounds for radial functions, and Theorem 4.4 reviews an integral bound of Gorbachev 33 that does not assume radial symmetry.

In Section 5 we present a unified solution to a class of previously scattered extremal problems. Specifically, Theorem5.1states that if $f$ is a probability density on $\mathbb{R}^{d}$ whose characteristic function $\varphi$ vanishes outside the unit ball, then

$$
\int|x|^{2} f(x) \mathrm{d} x=-\Delta \varphi(0) \geq 4 j_{(d-2) / 2}^{2}
$$

where $j_{\nu}$ denotes the first positive zero of the Bessel function $J_{\nu}$. Corollary 5.2 is a related minimum variance result for unimodal densities. The aforementioned bound is sharp and does not assume radial symmetry. However, a common feature of the proofs in Sections 4 and 5 is a reduction to radial functions, and the results allow for simplified proofs under the additional assumption of the existence of a Boas-Kac root. As we now know, a Boas-Kac factorization may fail to exist if $d \geq 2$, and our proofs resort instead to a more general representation of $\varphi$ as a possibly infinite sum of self-convolutions that is due to Rudin [57].

1.3. Applications. Our results have applications ranging from probability theory, time series analysis, and spatial statistics to optics, crystallography, and signal processing, and we sketch them below.

The optical transfer function is the frequency response of an optical system to light intensity distributions in the object plane [61. It is a continuous and positive definite function; hence, optimization problems translate into extremal problems for positive definite functions. The extended generalized Fejér-Riesz theorem in optics 59] claims that every compactly supported, radial positive definite function in $\mathbb{R}^{2}$ admits a Boas-Kac root. This is disproved by Theorem 3.1 and the examples that follow. However, Corollary 3.2 proves the existence of Boas-Kac roots in the practically important case of nonnegative functions, and the minimum-bias result in 59] remains valid, as we show in Section 5. Similar questions about convolution roots occur in small-angle scattering in crystallography 25, 26 and in phase retrieval problems [56 49].

Let $Z(y), y \in \mathbb{R}^{d}$, be a real-valued stationary Gaussian process with covariance function $\varphi(x), x \in \mathbb{R}^{d}$. Stationary Gaussian random fields play key roles in the statistical modeling of time series and spatial data, and in geostatistics they are typically assumed to be isotropic, meaning that $\varphi$ is a radially symmetric function. Furthermore, it is well-known that the class of covariance functions of stationary and isotropic random fields coincides with the class of radial positive definite functions. If $\varphi$ has a representation of the form $\varphi=u \widetilde{*} u$ with a real-valued function $u \in$ $L^{2}\left(\mathbb{R}^{d}\right)$, the process $Z(y), y \in \mathbb{R}^{d}$, admits a spatial moving average representation, namely

$$
Z(y)=\int u\left(y-y^{\prime}\right) \mathrm{d} W\left(y^{\prime}\right), \quad y \in \mathbb{R}^{d},
$$

where $W$ is a Gaussian white noise process. The real-valued convolution root $u$ is then called a dilution function. If the dilution function has compact support, the spatial moving average representation allows for the efficient simulation of the 
associated Gaussian random field [51, 18]. However, Chilès and Delfiner [14, p. 489] note that "it has not been proved, to our knowledge, that every covariance with bounded support can be related to a dilution function with bounded support".

This problem is studied in Sections 2 and 3. Part (b) of Theorem 2.1 shows that in dimension $d=1$ real-valued Boas-Kac roots always exist. In the spatial case, $d \geq 2$, our results are negative. By Theorem 3.1 real-valued Boas-Kac roots, and thereby spatial moving average representations with half-support dilution functions, do not exist in general. In Section 3.5 we discuss the important case of the so-called spherical correlation function,

$$
\varphi(x)=1-\frac{3}{2}|x|+\frac{1}{2}|x|^{3}, \quad x \in \mathbb{R}^{d}, \quad|x| \leq 1,
$$

and $\varphi(x)=0$ otherwise. A dilution function with half-support exists in dimensions $d=1$ and $d=3$, but not in dimension $d=2$. However, every stationary and isotropic Gaussian process with a compactly supported correlation function admits a representation as a finite or countable sum of independent moving average processes with half-support dilution functions. We intend to give details elsewhere.

\section{FACtORizations in Dimension $d=1$}

2.1. Factorization theorem. Our basic result for the one-dimensional case is stated in terms of the half-support (Boas-Kac) convolution representation

$$
\varphi(x)=u \widetilde{*} u(x)=\int u(y) \overline{u(y+x)} \mathrm{d} y, \quad x \in \mathbb{R},
$$

of a positive definite function with compact support. Its proof in Section 2.2 relies on the factorization

$$
f(z)=\psi(z) \psi^{*}(z), \quad z \in \mathbb{C},
$$

of the analytically continued associated density $f$ into the product of an entire function $\psi$, with half the type of $f$ and the appropriate additional properties, times its conjugate $\psi^{*}(z)=\overline{\psi(\bar{z})}$ obtained by reflection at the real line.

Theorem 2.1. Let $\tau$ be positive and finite, and let $\varphi(x), x \in \mathbb{R}$, be a continuous positive definite function with $\varphi(x)=0$ for $|x| \geq \tau$. Consider the analytic continuation $f(z), z \in \mathbb{C}$, of its inverse Fourier transform $f=\mathcal{F}^{-1} \varphi$ to an entire function of finite exponential type $\tau$.

(a) A Boas-Kac root exists: There exists a complex-valued function $u \in L^{2}(\mathbb{R})$ with $u(x)=0$ for $|x| \geq \tau / 2$ such that (1) holds.

(b) A real-valued Boas-Kac root exists if and only if $f$, or $\varphi$, is even.

(c) An even Boas-Kac root exists if and only if $f$ is even and satisfies conditions (A) and (B).

(d) A real-valued and even Boas-Kac root exists if and only if $f$ is even and satisfies conditions $(\mathrm{A}),(\mathrm{B})$, and $(\mathrm{C})$.

Statement (a) is the classical result of Boas and Kac 9]; statements (b), (c), and (d) are new. Our proof follows the lines of Boas [8] and avoids an incomplete half-type argument in [9]. Alternative proofs of statement (a) that are based on the Fejér-Riesz theorem have been given by Achieser [1], who gives credit to Krein, and by Garsia, Rodemich, and Rumsey [24]. Indeed, Krein [43] formulated statement (a) in terms of entire functions in a research announcement in 1940. Akutowicz [2], Walther [60], and Hofstetter [36] studied the nonuniqueness of the Boas-Kac root. 
If $\varphi$ is nonnegative, the Boas-Kac root can always be chosen as an even function; see Corollary 3.2 below. However, Example 2.3 shows that a nonnegative $\varphi$ does not necessarily admit a real-valued and even Boas-Kac root.

2.2. Proof of Theorem 2.1. It suffices to prove statements (b) through (d), and we start by showing the necessity of the respective conditions. Note that the existence of a real-valued and/or even Boas-Kac root $u$ implies that $\varphi$ is even. Indeed, if $u$ is even, then so is $\varphi=u \widetilde{*} u$. If $u$ is real-valued, then so is $\varphi=u \widetilde{*} u$, and since $\varphi$ is positive definite it is an even function. Hence, we may from now on assume that $\varphi$, hence $f$, is even.

The necessity of conditions (A), (B), or (C) in statements (c) and (d) follows from the equivalence of the Boas-Kac convolution representation (11) and the associated factorization (2), where

$$
u(x)=\frac{1}{(2 \pi)^{1 / 2}} \int_{\mathbb{R}} e^{-i x y} \psi(y) \mathrm{d} y, \quad x \in \mathbb{R},
$$

and

$$
\psi(z)=\frac{1}{(2 \pi)^{1 / 2}} \int_{\mathbb{R}} e^{i z x} u(x) \mathrm{d} x, \quad z \in \mathbb{C} .
$$

For ease of reference, we recall that $u$ is real-valued if and only if $\psi$ is Hermitian, i.e., $\psi^{*}(z)=\psi(-z) ; u$ is even if and only if $\psi$ is even, i.e., $\psi(z)=\psi(-z)$; and $u$ is real-valued and even if and only if $\psi^{*}(z)=\psi(z)=\psi(-z)$, for all $z \in \mathbb{C}$.

To prove the necessity of conditions (A) and (B) in parts (c) and (d), suppose that the Boas-Kac root $u$ is even. Then $\psi$ is also even, and $f(i y)=\psi(i y) \psi^{*}(i y)=$ $|\psi(i y)|^{2}$ for $y \in \mathbb{R}$. Hence $f$ is nonnegative on the imaginary axis which implies (B). Condition (A) holds because the even functions $\psi$ and $\psi^{*}$ vanish to the same even order at the origin. To prove the necessity of condition $(C)$ in statement (d), suppose that $u$ is real-valued and even. Then $f=\psi^{2}$, and the $f$-order of any zero is twice its $\psi$-order.

The key tool in the proof of the sufficiency of the conditions in parts (b), (c) and (d) is Hadamard's factorization theorem [20, p. 21]: Any nonzero entire function $g$ of finite exponential type admits a representation of the form

$$
g(z)=e^{\alpha z+\beta} z^{m} \prod_{k}\left(1-\frac{z}{\omega_{k}}\right) e^{z / \omega_{k}}
$$

where $\alpha$ and $\beta$ are complex constants, the $\omega_{k}$ are the zeros of $g$ away from the origin, and $m \geq 0$ is the multiplicity of the zero at the origin. Furthermore, the sequence $\omega_{k}$ satisfies

$$
\sum_{k}\left|\omega_{k}\right|^{-1-\delta}<\infty \text { for every } \delta>0 .
$$

We recall two more facts that hold whenever $g$ is bounded on the real axis; see 20 . pp. 22 and 23]. First, the series $\sum_{k}\left|\Im \omega_{k}^{-1}\right|$ is convergent. Second, the exact type $\tau$ of $g$ is attained along the imaginary axis and can be calculated as

$$
\tau=\max \left\{\limsup _{y \rightarrow \infty} \frac{\log |g(i y)|}{y}, \limsup _{y \rightarrow \infty} \frac{\log |g(-i y)|}{y}\right\} .
$$

We know that $f(z)=(2 \pi)^{-1} \int e^{-i z x} \varphi(x) \mathrm{d} x$ is an entire function of exponential type $\tau$. Since $\varphi$ is even, we have $f(z)=f(-z)=f^{*}(z)$ for $z \in \mathbb{C}$. Thus, $\alpha=0$ and the zeros $\omega_{k}$ in the Hadamard product (5) for $f$ lie symmetrically with respect to 
both the real and the imaginary axis. Moreover, $f(x) \geq 0$ for $x \in \mathbb{R}$ and hence the order of any real zero of $f$ is even. We write $a_{k}$ for the strictly positive real zeros; $i b_{k}$ for the purely imaginary zeros with strictly positive imaginary part; and $c_{k}$ for the zeros in the open first quadrant with strictly positive real and imaginary parts. Counting each $i b_{k}$ and $c_{k}$ according to its multiplicity, and each $a_{k}$ with half its multiplicity, we can write the Hadamard product of $f$ as

$$
f(z)=B z^{2 p} f_{r}(z) f_{i}(z) f_{c}(z), \quad z \in \mathbb{C},
$$

with a positive number $B$, a nonnegative integer $p$, and factors

$$
\begin{aligned}
& f_{r}(z)=\prod_{k}\left(1-\frac{z}{a_{k}}\right)^{2}\left(1+\frac{z}{a_{k}}\right)^{2}=\prod_{k}\left(1-\left(\frac{z}{a_{k}}\right)^{2}\right)^{2}, \\
& f_{i}(z)=\prod_{k}\left(1-\frac{z}{i b_{k}}\right)\left(1+\frac{z}{i b_{k}}\right)=\prod_{k}\left(1+\left(\frac{z}{b_{k}}\right)^{2}\right),
\end{aligned}
$$

and

$$
\begin{aligned}
f_{c}(z)= & \prod_{k}\left(1-\frac{z}{c_{k}}\right)\left(1-\frac{z}{\overline{c_{k}}}\right)\left(1+\frac{z}{c_{k}}\right)\left(1+\frac{z}{\overline{c_{k}}}\right) \\
= & \prod_{k}\left(1-\left(\frac{z}{c_{k}}\right)^{2}\right)\left(1-\left(\frac{z}{\overline{c_{k}}}\right)^{2}\right) .
\end{aligned}
$$

All other factors in (5) vanish or cancel each other, and uniform convergence on compact sets is guaranteed by (6). Since $f$ is bounded on the real line we have

$$
\sum_{k} b_{k}^{-1}<\infty \text { and } \sum_{k}\left|\Im c_{k}^{-1}\right|<\infty .
$$

In the following we assume without loss of generality that $B=1$.

The sufficiency of the conditions in parts (b), (c), and (d) is now proved by assembling the factor $\psi$ in (2) from suitable factors of $f$ in such a way that the respective properties hold. The proof that the products converge uniformly on compacts and that $\psi$ has exponential type $\tau / 2$ is deferred to the end of the section.

For part (b) put

$$
\psi(z)=(i z)^{p} e^{i \gamma z} \prod_{k}\left(1-\left(\frac{z}{a_{k}}\right)^{2}\right) \prod_{k}\left(1-\frac{z}{i b_{k}}\right) \prod_{k}\left(1-\frac{z}{c_{k}}\right)\left(1+\frac{z}{\overline{c_{k}}}\right) .
$$

By (9) and since $c_{k}^{-1}-\bar{c}_{k}^{-1}$ is purely imaginary, the exponential convergence generating factors for the complex zeros can, and have been, absorbed into the real constant $\gamma$. The factorization (2) holds, and $\psi(z)$ is Hermitian since each of the following factors is such: $g(z)=(i z)^{p} ; g(z)=e^{i \gamma z}$ where $\gamma \in \mathbb{R} ; g(z)=1-(z / a)^{2}$ where $a \in \mathbb{R} ; g(z)=1-z /(i b)$ where $b \in \mathbb{R} ;$ and $g(z)=(1-z / c)(1+z / \bar{c})$ where $c \in \mathbb{C}$.

With respect to part (c) suppose that $f$ satisfies (A) and (B). Define

$$
\psi(z)=z^{p} \prod_{k}\left(1-\left(\frac{z}{a_{k}}\right)^{2}\right) \prod_{k}\left(1+\left(\frac{z}{b_{k}}\right)^{2}\right) \prod_{k}\left(1-\left(\frac{z}{c_{k}}\right)^{2}\right)
$$

where $p$ is even and where the zeros $i b_{k}$ with $b_{k}>0$ are now counted with half their multiplicity. Then (2) holds and $\psi$ is even. 
As for part (d), suppose that $f$ satisfies (A), (B), and (C). Define

$$
\psi(z)=z^{p} \prod_{k}\left(1-\left(\frac{z}{a_{k}}\right)^{2}\right) \prod_{k}\left(1+\left(\frac{z}{b_{k}}\right)^{2}\right) \prod_{k}\left(1-\left(\frac{z}{c_{k}}\right)^{2}\right)\left(1-\left(\frac{z}{\overline{c_{k}}}\right)^{2}\right)
$$

where $p$ is even and where both the zeros $i b_{k}$ with $b_{k}>0$ and the zeros $c_{k}$ in the open first quadrant are counted with half their multiplicity. Then (2) holds and $\psi$ is Hermitian and even.

To complete the proof we need to show that the function $\psi$ defined by (10), (11), or (12), respectively, is entire of exponential type $\tau / 2$. The zeros of $\psi$ form a subset of the zeros of $f$, and (6) and (9) imply that the defining product converges uniformly on compacts. Thus $\psi$ is an entire function, and by (6) and Theorem 2.6.5 in 8 its order is 1 . It remains to be shown that $\psi$ has exponential type $\tau / 2$. If $\psi$ is defined as in (11) or (12), then $|f|=|\psi|^{2}$ both on the imaginary and on the real axis. We may therefore apply (7) and it follows that the type of $\psi$ is $\tau / 2$. If $\psi$ is defined as in (10) with a suitably chosen real constant $\gamma$, its type is $\tau / 2$ by Theorem 7.5.1 in 8. It suffices to note that $\psi$ has all its zeros in the closed upper half-plane and is constructed in the same way as in the reference. The proof of Theorem 2.1 is complete.

2.3. Remarks. The factorizations of $f$ constructed in the proof are not necessarily unique. In (10) we can replace any $b_{k}$ by $-b_{k}$ and any $c_{k}$ by $-c_{k}$, and in (11) we can replace any $c_{k}$ by $\overline{c_{k}}$. See Akutowicz [2, Walther [60, and Hofstetter [36] for a discussion of "zero flipping" and "phase retrieval." However, if a real-valued and even Boas-Kac root exists then it is unique up to sign and given by

$$
u=(2 \pi)^{d / 2} \mathcal{F}_{d}^{-1} f^{1 / 2}
$$

which is a well-known result in crystallography; see 25] and references therein. Indeed, (12) does not allow for zero flipping. This suggests a straightforward numerical test for the existence of a real-valued and even Boas-Kac root. Given an even characteristic function $\varphi$ with $\varphi(x)=0$ for $|x| \geq \tau$, find its inverse Fourier transform $f$ and compute $\mathcal{F} f^{1 / 2}$. If $\mathcal{F} f^{1 / 2}(x) \neq 0$ for some $x>\tau / 2$, then $\varphi$ does not admit a real-valued and even Boas-Kac root. A difficulty with this test is that $f^{1 / 2}$ is square-integrable but not necessarily integrable. Because the Fourier transform $\mathcal{F} f^{1 / 2}$ may not be well-defined pointwise, we instead estimate its integral over small intervals, using the following lemma.

Lemma 2.2. Let $g \in L_{2}(\mathbb{R})$ be a real-valued, even, and differentiable function such that $\int_{A}^{\infty}\left|g^{\prime}(y)\right| \mathrm{d} y<\infty$ for $A>0$. Then for all $x_{2}>x_{1}>0$ and $A>0$ one has

$$
\left|\int_{x_{1}}^{x_{2}} \mathcal{F} g(x) \mathrm{d} x-2 \int_{0}^{A} \frac{\sin \left(x_{2} y\right)-\sin \left(x_{1} y\right)}{y} g(y) \mathrm{d} y\right| \leq 4 \log \frac{x_{2}}{x_{1}} \int_{A}^{\infty}\left|g^{\prime}(y)\right| \mathrm{d} y .
$$

Proof. The Fourier transform $\mathcal{F} g(x)$ may be defined as the $L^{2}(\mathbb{R})$ limit of the clipped Fourier integrals $v_{A}(x)=\int_{-A}^{A} \cos (x y) g(y) \mathrm{d} y$ as $A \rightarrow \infty$. We fix $x>0$ and study the asymptotic behavior of $v_{A}(x)$ as $A \rightarrow \infty$. Let $0<A<B$. Partial integration and a straightforward estimate give

$$
\frac{1}{2}\left|v_{B}(x)-v_{A}(x)\right| \leq \frac{1}{x}\left(|g(A)|+|g(B)|+\int_{A}^{B}\left|g^{\prime}(y)\right| \mathrm{d} y\right) .
$$


Note that $\lim _{y \rightarrow \infty} g(y)=0$ since $g \in L^{2}(\mathbb{R})$ and $\int_{A}^{\infty}\left|g^{\prime}(y)\right| \mathrm{d} y<\infty$ for all $A>0$. Hence, $\left(v_{A}(x)\right)_{A>0}$ is a Cauchy net and $v(x)=\lim _{A \rightarrow \infty} v_{A}(x)$ exists pointwise for all $x \neq 0$. Fixing $A>0$ and taking the limit as $B \rightarrow \infty$ in (15) shows that

$$
\left|v(x)-v_{A}(x)\right| \leq \frac{2}{x}\left(|g(A)|+\int_{A}^{\infty}\left|g^{\prime}(y)\right| \mathrm{d} y\right) \leq \frac{4}{x} \int_{A}^{\infty}\left|g^{\prime}(y)\right| \mathrm{d} y .
$$

Since pointwise and $L^{2}(\mathbb{R})$ limits coincide we have $\mathcal{F} g(x)=v(x)$ for almost all $x \in \mathbb{R}$. The inequality (14) follows easily by integration of (16) and an application of Fubini's theorem.

The numerical test is performed by putting $g=f^{1 / 2}$ in (14), computing the main term $I$ and the error term $E$ given by

$$
I=\int_{0}^{A} \frac{\sin \left(x_{2} y\right)-\sin \left(x_{1} y\right)}{y} f^{1 / 2}(y) \mathrm{d} y
$$

and

$$
E=\log \frac{x_{2}}{x_{1}} \int_{A}^{\infty} \frac{\left|f^{\prime}(y)\right|}{f^{1 / 2}(y)} \mathrm{d} y
$$

respectively, and checking whether

$$
|I|>E \quad \text { for some } \quad x_{2}>x_{1} \geq \tau / 2 \text { and } A>0 .
$$

If this is the case, then the first term in (14) does not vanish, and a real-valued and even Boas-Kac root does not exist. Note that if $f(x)$ is nonincreasing for $x>A$, then the integral in the error term $E$ equals $2 f^{1 / 2}(A)$. Evidently, the test applies to any suitably normalized version of $f$.

2.4. Examples. The conditions (A), (B), and (C) in Theorem 2.1 are logically independent of each other and may occur in any combination. In fact, all possible cases are covered by the class of characteristic functions $\varphi$ whose densities $f=\mathcal{F}^{-1} \varphi$ have analytic continuation

$$
f(z)=c z^{2 p}\left(z^{2}+1\right)^{q}\left(z^{4}+4\right)^{r}\left(\frac{1-\cos z}{z^{2}}\right)^{s} .
$$

Here $p, q$, and $r$ are nonnegative integers, $s$ is a positive integer greater than $p+q+2 r$, and the proportionality constant $c$ is chosen such that $f$ is a probability density on $\mathbb{R}$. Then $f(z)$ is an even entire function of exponential type $s$ with real zeros at multiples of $2 \pi$. The zero at the origin has order $2 p$, and since

$$
2 \frac{1-\cos z}{z^{2}}=\left(\frac{\sin (z / 2)}{z / 2}\right)^{2}=\prod_{k=1}^{\infty}\left(1-\frac{z^{2}}{(2 k \pi)^{2}}\right)^{2}
$$

all other real zeros have order $2 s$. If purely imaginary zeros exist, then they are at $\pm i$ with multiplicity $q$. Zeros that are neither real nor purely imaginary exist if and only if $r>0$, and then they are at $\pm 1 \pm i$ with multiplicity $r$. Hence condition (A), (B), or (C) is satisfied if and only if $p, q$, or $r$, respectively, is even. 

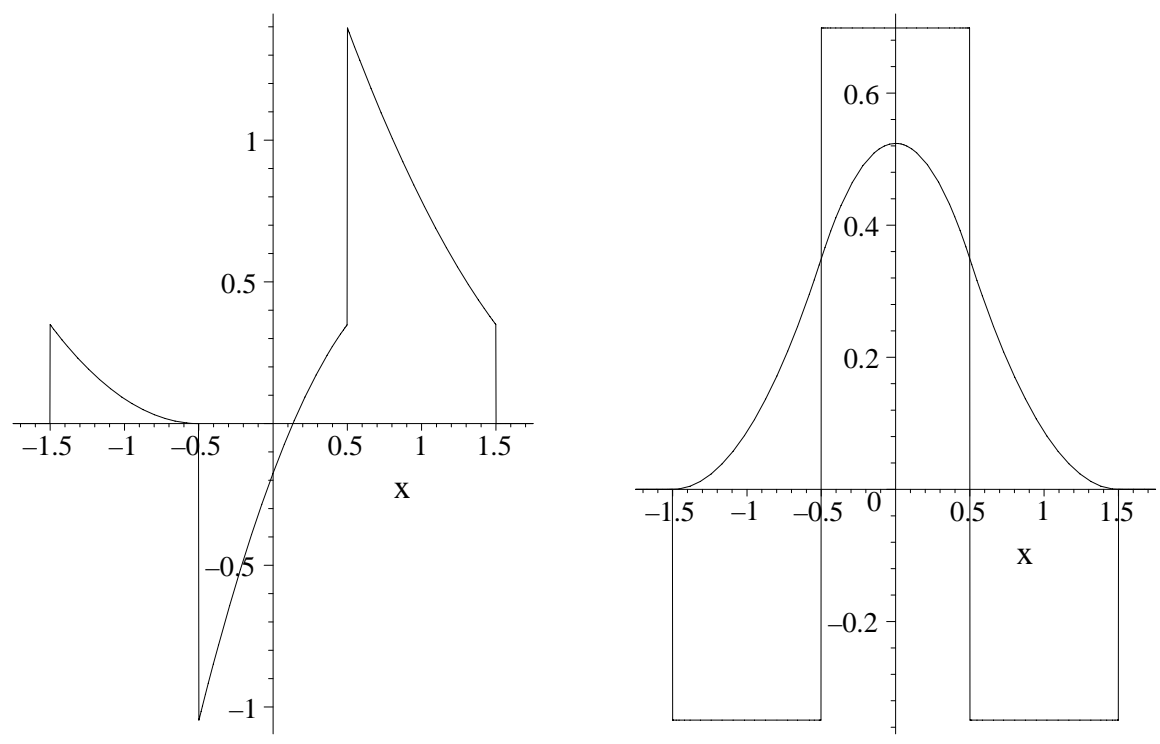

Figure 1. The real-valued Boas-Kac root $u_{1}$ (left) and the real and imaginary parts of the even Boas-Kac root $u_{2}$ (right) in Example 2.3

Example 2.3. The positive definite function defined by

$$
\varphi(x)= \begin{cases}1-\frac{1}{123}\left(150|x|+30 x^{2}-15 x^{4}+5|x|^{5}\right), & |x| \leq 1, \\ -\frac{1}{246}\left(219-225|x|+210 x^{2}-150|x|^{3}+45 x^{4}-5|x|^{5}\right), & 1 \leq|x| \leq 2, \\ \frac{1}{246}(3-|x|)\left(111-108|x|+54 x^{2}-12|x|^{3}+x^{4}\right), & 2 \leq|x| \leq 3,\end{cases}
$$

and $\varphi(x)=0$ for $|x| \geq 3$ corresponds to the choice $p=0, q=0, r=1$, and $s=3$ in (18). Condition $(\mathrm{C})$ is violated while $(\mathrm{A})$ and $(\mathrm{B})$ hold. Figure 1 illustrates a real-valued Boas-Kac root given by

$$
u_{1}(x)=\frac{\sqrt{205}}{164} \begin{cases}1+4 x+4 x^{2}, & -3 / 2<x<-1 / 2, \\ -2+16 x-8 x^{2}, & -1 / 2<x<1 / 2, \\ 25-20 x+4 x^{2}, & 1 / 2<x<3 / 2\end{cases}
$$

and $u_{1}(x)=0$ for $|x| \geq 3 / 2$, and an even Boas-Kac root defined by

$$
u_{2}(x)=\frac{\sqrt{205}}{164} \begin{cases}(3+2 x)^{2}-4 i, & -3 / 2<x<-1 / 2, \\ 6-8 x^{2}+8 i, & -1 / 2<x<1 / 2, \\ (2 x-3)^{2}-4 i, & 1 / 2<x<3 / 2,\end{cases}
$$

and $u_{2}(x)=0$ for $|x| \geq 3 / 2$. However, a Boas-Kac root that is both real-valued and even does not exist. 
Example 2.4. The density associated with the characteristic function

$$
\varphi(x)= \begin{cases}1-\frac{5}{4}|x|, & 0 \leq|x| \leq 1 \\ -\frac{1}{2}+\frac{1}{4}|x|, & 1 \leq|x| \leq 2\end{cases}
$$

and $\varphi(x)=0$ for $|x| \geq 2$ is $f(x)=(2-\cos x)(1-\cos x) /\left(2 \pi x^{2}\right)$. The nonreal zeros of its analytic continuation $f(z)$ are simple and at $z=2 \pi n \pm i y$, with $n$ an integer and $y$ the positive root of the equation $\cosh (y)=2$. Conditions $(\mathrm{B})$ and $(\mathrm{C})$ are violated, and an even Boas-Kac root does not exist.

Example 2.5. The characteristic function $\varphi(x)=(1-|x|)_{+}^{2}, x \in \mathbb{R}$, has density $f(x)=2(x-\sin x) /\left(\pi x^{3}\right), x \in \mathbb{R}$. Conditions (A) and (B) hold, but it is difficult to check analytically whether condition $(\mathrm{C})$ is satisfied. However, $f$ decreases on the positive half-axis and we may apply the numerical test (17). Putting $x_{1}=1 / 2$, $x_{2}=11 / 20$, and $A=40$ yields values of about 0.0116 and 0.0038 for the main term $I$ and the error term $E$, respectively. Hence, $\varphi$ does not admit a real-valued and even Boas-Kac root.

\section{FACTORIZATION OF RADIAL FUNCTIONS IN DIMENSION $d \geq 2$}

3.1. Factorization theorem. A function $g(x)$ on $\mathbb{R}^{d}$ is radial or radially symmetric if it depends on the Euclidean norm $|x|$ only. We denote its radial part by $g_{0}$, i.e., $g(x)=g_{0}(|x|)$ for $x \in \mathbb{R}^{d}$, and we put $g_{0}(t)=g_{0}(-t)$ for $t<0$ so that $g_{0}$ is an even function on $\mathbb{R}$. A function $g$ defined on $\mathbb{C}^{d}$ is said to be radial if its restriction to $\mathbb{R}^{d}$ is such.

Let $\varphi$ be a positive definite function on $\mathbb{R}^{d}$ with $\varphi(x)=0$ if $|x| \geq \tau$. We assume that $\varphi(x)=\varphi_{0}(|x|)$ is radial, hence real-valued. As in the one-dimensional case we seek representations of the form

$$
\varphi(x)=u \widetilde{*} u(x)=\int u(y) \overline{u(y+x)} \mathrm{d} y, \quad x \in \mathbb{R}^{d},
$$

where $u$ is a generally complex-valued, square-integrable function on $\mathbb{R}^{d}$ with $u(x)=$ 0 if $|x| \geq \tau / 2$. We call any function $u$ satisfying these conditions a Boas-Kac root of $\varphi$. Note that $u$ is not required to be radially symmetric.

The $d$-dimensional inverse Fourier transform $f=\mathcal{F}_{d}^{-1} \varphi$ of $\varphi$ satisfies $0 \leq f \in$ $L^{1}\left(\mathbb{R}^{d}\right)$ and represents the density associated with $\varphi$. Clearly, $f$ is radial, and it admits an analytical continuation to an entire function on $\mathbb{C}^{d}$ given by

$$
f(\zeta)=\frac{1}{(2 \pi)^{d}} \int_{\mathbb{R}^{d}} e^{-i \zeta \cdot x} \varphi(x) \mathrm{d} x, \quad \zeta \in \mathbb{C}^{d},
$$

where $\xi \cdot \eta=\sum_{l=1}^{d} \xi_{l} \eta_{l}$ for vectors $\xi=\left(\xi_{1}, \ldots, \xi_{d}\right)$ and $\eta=\left(\eta_{1}, \ldots, \eta_{d}\right)$ in $\mathbb{C}^{d}$. The radial part $f_{0}$ of $f$ satisfies $f_{0}(t)=f(t, 0, \ldots, 0)$ for $t \in \mathbb{R}$. Hence $f_{0}(z)=$ $f(z, 0, \ldots, 0), z \in \mathbb{C}$, is its continuation to an entire function on the complex plane. Since $f_{0}$ is even, there exists an entire function $F$ such that $f_{0}(z)=F\left(z^{2}\right)$ for $z \in \mathbb{C}$. Then $f(x)=f_{0}(|x|)=F(x \cdot x)$ for $x \in \mathbb{R}^{d}$, and since $f(\zeta)$ is everywhere holomorphic by (20) this relationship extends coordinatewise to the complex domain. Therefore, the relations between $f, f_{0}$, and $F$ may be summarized as follows,

$$
f(\zeta)=F(\zeta \cdot \zeta), \quad \zeta \in \mathbb{C}^{d} ; \quad f(z, 0, \ldots, 0)=f_{0}(z)=F\left(z^{2}\right), \quad z \in \mathbb{C} .
$$


Straightforward estimates using (21) and (20) show that $f_{0}$ is an entire function of exponential type $\tau$, and that $f$ is an entire function of radial exponential Ptype $\tau$, in the following sense. An entire function $g$ on $\mathbb{C}^{d}$ is said to be of radial exponential P-type $\rho$ if (i) it has finite exponential type on $\mathbb{C}^{d}$, i.e., if $|g(\zeta)| \leq$ $A \exp \left(a\left(\left|\zeta_{1}\right|+\ldots+\left|\zeta_{d}\right|\right)\right)$ for all $\zeta \in \mathbb{C}^{d}$, where $A>0, a>0$ are finite constants; and if (ii) for any pair of vectors $x, y \in \mathbb{R}^{d}$ with $|y|=1$ one has

$$
\limsup _{r \rightarrow \infty} r^{-1} \log |g(x+i r y)| \leq \rho .
$$

This notion of exponential type differs from the usual one used in the previous section. It refers to the Plancherel-Pólya extension of the Paley-Wiener theorem, which connects the growth indicator of an entire function on $\mathbb{C}^{d}$ to the support of its Fourier transform [55]. Here we require only the special case in which the support is contained in a ball around the origin. The Plancherel-Pólya theorem then asserts the following: Let $\rho>0$. Then $g(\zeta), \zeta \in \mathbb{C}^{d}$, is an entire function of radial exponential P-type $\rho$ with $g \in L^{2}\left(\mathbb{R}^{d}\right)$ if and only if

$$
g(\zeta)=\frac{1}{(2 \pi)^{d}} \int_{\mathbb{R}^{d}} e^{-i \zeta \cdot x} \gamma(x) \mathrm{d} x
$$

with a function $\gamma \in L^{2}\left(\mathbb{R}^{d}\right)$ such that $\gamma(x)=0$ if $|x| \geq \rho$. Note that $g$ is not required to be radial. Returning to $\varphi$ and $f$ the following equivalence holds for such function pairs: A Boas-Kac root $u$ of $\varphi$ exists if and only if there is an entire function $\psi$ of radial exponential P-type $\tau / 2$ such that $\psi \in L^{2}\left(\mathbb{R}^{d}\right)$ and

$$
f(\zeta)=\psi(\zeta) \psi^{*}(\zeta), \quad \zeta \in \mathbb{C}^{d},
$$

where $\psi^{*}(\zeta)=\overline{\psi(\bar{\zeta})}$. We call any representation of the form (24) with a function $\psi$ satisfying these conditions a Boas-Kac factorization of $f$. The relationship between the Boas-Kac root $u$ and the Boas-Kac factor $\psi$ is then given by

$$
u(x)=\frac{1}{(2 \pi)^{d / 2}} \int_{\mathbb{R}^{d}} e^{-i x \cdot y} \psi(y) \mathrm{d} y, \quad x \in \mathbb{R}^{d},
$$

and

$$
\psi(\zeta)=\frac{1}{(2 \pi)^{d / 2}} \int_{\mathbb{R}^{d}} e^{i \zeta \cdot x} u(x) \mathrm{d} x, \quad \zeta \in \mathbb{C}^{d} .
$$

We now state the analogue of Theorem 2.1 in the higher-dimensional radial case. The respective conditions pertain to the zeros of $f_{0}$.

Theorem 3.1. Let $\varphi$ be a continuous radial positive definite function on $\mathbb{R}^{d}$ with $\varphi(x)=0$ for $|x| \geq \tau$. Consider the analytic continuation $f_{0}(z), z \in \mathbb{C}$, of the radial part of the density $f=\mathcal{F}_{d}^{-1} \varphi$ associated with $\varphi$.

(a) If $d=2$, a Boas-Kac root exists if and only if $f_{0}$ satisfies (B). The BoasKac root can be chosen as a radial function if and only if $f_{0}$ satisfies (A) and $(\mathrm{B})$.

(b) If $d \geq 3$, a Boas-Kac root exists if and only if $f_{0}$ satisfies (A) and (B). Furthermore, any Boas-Kac root is necessarily a radial function up to a translation.

(c) If $d \geq 2$, a real-valued Boas-Kac root exists if and only if $f_{0}$ satisfies conditions $(\mathrm{A}),(\mathrm{B})$, and $(\mathrm{C})$. Furthermore, any real-valued Boas-Kac root is necessarily a radial function up to a translation. 
The phrase "radial up to a translation" in statements (b) and (c) means that there exists a $\mu \in \mathbb{R}^{d}$ such that the shifted Boas-Kac root $u(x+\mu)$ is a function of $|x|$ only. If $f_{0}$ has exact exponential type $\tau$, i.e., if $[-\tau, \tau]$ is the smallest interval that contains the support of $\varphi_{0}$, the translation is necessarily trivial. In contrast to the one-dimensional case and contrary to earlier claims in the literature [59], a Boas-Kac root may not exist if $d>1$. We give explicit counterexamples below. However, there is a large class of functions that are of particular importance in applications and for which Boas-Kac roots exist.

Corollary 3.2. Let $d \geq 1$. Suppose that $\varphi$ satisfies the assumptions of Theorem 3.1 and is furthermore nonnegative. Then a Boas-Kac root exists and it can be chosen as a radial function.

Proof. If $\varphi$ is nonnegative, then $f(i y), y \in \mathbb{R}^{d}$, is the Laplace transform of the nonnegative measure $\varphi(x) \mathrm{d} x$. Hence, $f_{0}$ is strictly positive along the imaginary axis and satisfies conditions (A) and (B). The corollary then follows from part (c) of Theorem 2.1 if $d=1$, from part (a) of Theorem 3.1 if $d=2$, and from part (b) of Theorem 3.1 if $d \geq 3$.

The proof of Theorem 3.1 is given in Section 3.2 and relies on the analytical tools introduced above. An alternative construction of a radial Boas-Kac root under conditions (A) and (B) makes use of the Radon transform and is given in Section 3.3 along with other remarks. Sections 3.4 and 3.5 provide examples and applications, and Section 3.6 discusses related results.

3.2. Proof of Theorem 3.1 Similar to the case $d=1$, the proof depends on the equivalence of the Boas-Kac convolution representation (19) for the positive definite function $\varphi$ to the Boas-Kac factorization (24) for the density $f=\mathcal{F}_{d}^{-1} \varphi$, where the Boas-Kac root $u$ and the factor $\psi$ are connected as in (25). We first prove the sufficiency of the conditions in parts (a), (b), and (c).

As seen above, the radial part $f_{0}$ of $f$ is an even entire function of exponential type $\tau$ and therefore admits the factorization (8). In view of (21) we obtain the representation $f_{0}(z)=F\left(z^{2}\right)$ where

$$
F(w)=B w^{p} \prod_{k}\left(1-\frac{w}{a_{k}^{2}}\right)^{2} \prod_{k}\left(1+\frac{w}{b_{k}^{2}}\right) \prod_{k}\left(1-\frac{w}{c_{k}^{2}}\right)\left(1-\frac{w}{{\overline{c_{k}}}^{2}}\right)
$$

is an entire function of $w \in \mathbb{C}$, and where all quantities involved have the same meaning and the same multiplicities as in (8).

Suppose first that $f_{0}$ satisfies conditions (A) and (B). Then $p=2 q$ is even and we can define a subproduct of $F(w)$ in analogy to (11) by setting

$$
\chi(w)=B^{1 / 2} w^{q} \prod_{k}\left(1-\frac{w}{a_{k}^{2}}\right) \prod_{k}\left(1+\frac{w}{b_{k}^{2}}\right) \prod_{k}\left(1-\frac{w}{c_{k}^{2}}\right)
$$

where we count the $b_{k}$ factors with half their original multiplicity. Then

$$
F(w)=\chi(w) \chi^{*}(w), \quad w \in \mathbb{C},
$$

and as shown in the proof of Theorem $2.1 z \rightarrow \chi\left(z^{2}\right)$ is an even entire function of exponential type $\tau / 2$. Therefore, $\chi(w), w \in \mathbb{C}$, is of order $1 / 2$ and type $\tau / 2$, i.e.,

$$
\limsup _{r \rightarrow \infty} r^{-1 / 2} \log \max _{|w|=r}|\chi(w)| \leq \tau / 2
$$


The factorization (27) is lifted to $d$ dimensions by putting $\psi(\zeta)=\chi(\zeta \cdot \zeta)$ for $\zeta \in \mathbb{C}^{d}$, so that

$$
f(\zeta)=F(\zeta \cdot \zeta)=\chi(\zeta \cdot \zeta) \chi^{*}(\zeta \cdot \zeta)=\psi(\zeta) \psi^{*}(\zeta), \quad \zeta \in \mathbb{C}^{d} .
$$

The function $\psi$ is entire and of radial exponential P-type $\tau / 2$. Indeed, by (28) and the estimate

$$
|\zeta \cdot \zeta|^{1 / 2} \leq\left(|x|^{2}+|y|^{2}\right)^{1 / 2} \leq\left|\zeta_{1}\right|+\cdots+\left|\zeta_{d}\right|, \quad \zeta=x+i y, \quad x, y \in \mathbb{R}^{d},
$$

it has finite exponential type on $\mathbb{C}^{d}$; if $\zeta_{r}=x+$ iry with $x, y \in \mathbb{R}^{d}$ and $|y|=1$, then the modulus of $\zeta_{r} \cdot \zeta_{r}$ behaves like $r^{2}(1+o(1))$ as $r \rightarrow \infty$, and since

$$
\left|\psi\left(\zeta_{r}\right)\right|=\left|\chi\left(\zeta_{r} \cdot \zeta_{r}\right)\right| \leq \max _{|w|=\left|\zeta_{r} \cdot \zeta_{r}\right|}|\chi(w)|,
$$

we conclude from (28) that

$$
\limsup _{r \rightarrow \infty} r^{-1} \log \left|\psi\left(\zeta_{r}\right)\right| \leq \limsup _{r \rightarrow \infty}\left|\zeta_{r} \cdot \zeta_{r}\right|^{-1 / 2} \log \max _{|w|=\left|\zeta_{r} \cdot \zeta_{r}\right|}|\chi(w)| \leq \tau / 2 .
$$

Evidently $\psi$ is radial and square-integrable on $\mathbb{R}^{d}$ and so is the Boas-Kac root $u$ in (25). We have shown that if $d \geq 2$, then conditions (A) and (B) are sufficient for the existence of a radial Boas-Kac root.

The proof of the sufficiency of conditions (A), (B), and (C) in statement (c) is very similar. The only difference is that the factors $1-w / c_{k}^{2}$ in the product defining $\chi(w)$ need to be replaced by $\left(1-w / c_{k}^{2}\right)\left(1-w /{\overline{c_{k}}}^{2}\right)$ and counted with half the original multiplicity of $c_{k}$. It then suffices to note that the resulting function $z \rightarrow \chi\left(z^{2}\right)$ is even and Hermitian.

As for the sufficiency part in statement (a) suppose that $d=2$ and $f_{0}$ satisfies condition (B). By (21) and (26) we may write

$$
f(\zeta)=F(\zeta \cdot \zeta)=(\zeta \cdot \zeta)^{p} G(\zeta \cdot \zeta), \quad \zeta=\left(\zeta_{1}, \zeta_{2}\right) \in \mathbb{C}^{2}
$$

where $G(w)=F(w) / w^{p}, w \in \mathbb{C}$. If condition (A) does not hold, then $p$ is odd and it is clear from the above that the factor $(\zeta \cdot \zeta)^{p}$ presents the only obstacle for a Boas-Kac factorization of $f$. However, if we define $Q(\zeta)=\left(\zeta_{1}+i \zeta_{2}\right)^{p}$, then $(\zeta \cdot \zeta)^{p}=Q(\zeta) Q^{*}(\zeta)$, and the existence of a Boas-Kac factorization follows.

The key result in the proof of the necessity of the respective conditions is the following theorem of Lawton [45].

Theorem 3.3 (Lawton). Let $d>1$. Let $\psi \in L^{2}\left(\mathbb{R}^{d}\right)$ be such that its Fourier transform has compact support and its modulus $|\psi|$ is radial and different from the null function. Then $\psi$ can be extended to an entire function on $\mathbb{C}^{d}$ given by

$$
\psi(\zeta)=A e^{i \mu \cdot \zeta} P(\zeta) R(\zeta \cdot \zeta), \quad \zeta \in \mathbb{C}^{d},
$$

with a nonzero complex constant $A ; \mu \in \mathbb{R}^{d} ; P$ a polynomial that for $d=2$ is of the form $\left(\zeta_{1}+i \zeta_{2}\right)^{k}\left(\zeta_{1}-i \zeta_{2}\right)^{l}$ and for $d \geq 3$ is of the form $(\zeta \cdot \zeta)^{m}$, with nonnegative integers $k, l$, or $m$; and $R(w)=\prod_{k}\left(1-w / \lambda_{k}^{2}\right), w \in \mathbb{C}$, with an infinite sequence of nonzero complex numbers $\lambda_{k}$ such that the product converges uniformly on compacts and $z \rightarrow R\left(z^{2}\right)$ is an entire function of finite exponential type.

The possibly different form of the polynomial $P$ in dimension 2 causes the distinction between the cases $d=2$ and $d \geq 3$.

Suppose then that a Boas-Kac factorization (24) of the density $f$ associated with $\varphi$ exists. Then $f(x)=|\psi(x)|^{2}$ for $x \in \mathbb{R}^{d}$, so that $|\psi|$ is radial. Therefore, 
Lawton's theorem applies and $\psi(\zeta)$ can be written as in (29). This representation is continually made use of in the following.

To prove the necessity of condition (B) for the existence of a Boas-Kac root, for every $d \geq 2$, consider any purely imaginary zero $i b$ of $f_{0}$, with $b \in \mathbb{R}, b \neq 0$. Let $\beta=(b, 0, \ldots, 0) \in \mathbb{C}^{d}$. Then $0=|f(i \beta)|=|\psi(i \beta) \psi(-i \beta)|$ and since $P(i \beta) \neq 0$ in (29), we must have $\lambda_{k}^{2}=-b^{2}$ for some $k$. The factor $1+(\zeta \cdot \zeta) / b^{2}$ then appears in both $\psi(\zeta)$ and $\psi^{*}(\zeta)$. Hence, it appears with even multiplicity in the product representation of $f$. The necessity of condition (B) follows by (21).

As for the necessity of condition (A), note that it is satisfied whenever the polynomial factor in (29) is of the form $P(\zeta)=(\zeta \cdot \zeta)^{m}$. Indeed, in this case $f(\zeta)=\psi(\zeta) \psi^{*}(\zeta)=F(\zeta \cdot \zeta)$ contains the factor $\zeta \cdot \zeta$ with even multiplicity, and the order of any zero of $f_{0}(z)=F\left(z^{2}\right)$ at the origin is a multiple of 4 . If $d \geq 3$, then $P$ is always of the above form and the existence of a Boas-Kac root implies (A). If $d=2$ and the Boas-Kac root $u$, hence $\psi$, is radially symmetric, the same holds. It remains to consider the case in which $d=2$ and a real-valued Boas-Kac root $u$ exists. Similar to the case $d=1$ we then have $\psi^{*}(\zeta)=\psi(-\zeta)$ for $\zeta \in \mathbb{C}^{2}$. Suppose that the polynomial factor of $\psi$ is of the form $P(\zeta)=\left(\zeta_{1}+i \zeta_{2}\right)^{k}\left(\zeta_{1}-i \zeta_{2}\right)^{l}$ with $k \neq l$. Extracting the factor $(\zeta \cdot \zeta)^{m}$ where $m=\min \{k, l\}$, we may assume that $P(\zeta)=\left(\zeta_{1}+i \zeta_{2}\right)^{n}$ for some positive integer $n$. This form of $P$ is incompatible with the assumption that $\psi^{*}(\zeta)=\psi(-\zeta)$ for $\zeta \in \mathbb{C}^{2}$. Indeed, if $\zeta_{1}=i \zeta_{2}$ and $0<\left|\zeta_{1}\right|<\inf _{k}\left|\lambda_{k}\right|$, then $P^{*}(\zeta)=\left(\zeta_{1}-i \zeta_{2}\right)^{n}=0$, hence $\psi^{*}(\zeta)=0$ whereas $\psi(-\zeta) \neq 0$. The contradiction shows that once again $P$ takes the form $P(\zeta)=(\zeta \cdot \zeta)^{m}$. Thus, condition (A) holds in all relevant cases.

To show the necessity of condition (C) in statement (c), suppose there exists a real-valued Boas-Kac root $u$ of $\varphi$. Consider any zero $c$ of $f_{0}$ that is neither real nor purely imaginary and let $\gamma=(c, 0, \ldots, 0) \in \mathbb{C}^{d}$. Then $0=f_{0}(c)=f(\gamma)=$ $\psi(\gamma) \psi(-\gamma)$ since $\psi^{*}(\zeta)=\psi(-\zeta)$. By (29), $|\psi( \pm \gamma)|=|A||P( \pm \gamma)|\left|R\left(c^{2}\right)\right|$ with $A P( \pm \gamma) \neq 0$, so we must have $R\left(c^{2}\right)=0$, and hence $\lambda_{k}=c$ or $\lambda_{k}=-c$ for some $k$. Therefore, the product representation of $f_{0}(z)=\psi(z, 0, \ldots, 0) \psi(-z, 0, \ldots, 0)$ contains the factor $1-(z / c)^{2}=(1-z / c)(1+z / c)$ with even multiplicity, and it follows that $(\mathrm{C})$ holds.

The second assertion in statements (b) and (c) follows easily from what has already been shown. It suffices to note that the effect of the factor $e^{i \mu \cdot \zeta}$ in (29) on the Boas-Kac root $u=(2 \pi)^{d / 2} \mathcal{F}_{d}^{-1} \psi$ is simply a translation, and that it is the only factor of $\psi$ that is possibly nonradial if $d \geq 3$, or if $d=2$ and $u$ is real-valued. The proof of Theorem [3.1] is complete.

3.3. Applications of the Radon transform. The Radon transform provides a useful complement to the complex variables methods applied so far. Here we only consider radial functions $g \in L^{1}\left(\mathbb{R}^{d}\right)$, in which case the Radon transform $\mathcal{R}_{d} g$ is defined as the even function on $\mathbb{R}$ given by

$$
\mathcal{R}_{d} g(t)=\sigma_{d-1} \int_{|t|}^{\infty} g_{0}(u)\left(u^{2}-t^{2}\right)^{(d-3) / 2} u \mathrm{~d} u
$$

where $\sigma_{k}=2 \pi^{k / 2} / \Gamma(k / 2)$ denotes the surface area of the unit sphere $\mathbb{S}^{k-1}$ in $\mathbb{R}^{k}[50$. pp. 22-23]. The Radon transform is connected to the Fourier transform through the relation $\mathcal{F}_{d} g(x)=\mathcal{F}_{1} \mathcal{R}_{d} g(|x|), x \in \mathbb{R}^{d}$, that is known as the projection theorem in computer tomography [50, p. 11]. In probabilistic terms, the Radon transform maps a radial density to its one-dimensional marginal density. 
As an application, we give an alternative construction of a radially symmetric Boas-Kac root under conditions (A) and (B). Consider a radial positive definite function $\varphi$ with density $f(x)=f_{0}(|x|), x \in \mathbb{R}^{d}$. Then

$$
f_{0}(|x|)=f(x)=\mathcal{F}_{d}^{-1} \varphi(x)=(2 \pi)^{-d} \mathcal{F}_{d} \varphi(x)=(2 \pi)^{-d} \mathcal{F}_{1} \mathcal{R}_{d} \varphi(|x|)
$$

since $\mathcal{F}_{d}^{-1} g=(2 \pi)^{-d} \mathcal{F}_{d} g$ for even, and hence for radial, functions $g$. By (B30), the support condition $\varphi(x)=0$ for $|x| \geq \tau$ implies that $\mathcal{R}_{d} \varphi(t)=0$ for $|t| \geq$ $\tau$. Since $\mathcal{F}_{1}^{-1}\left\{(2 \pi)^{d} f_{0}\right\}=\mathcal{R}_{d} \varphi$, we conclude that $\mathcal{F}_{1} f_{0}(t)$ vanishes for $|t| \geq \tau$. Furthermore, $f_{0}$ is nonnegative, even, and integrable on $\mathbb{R}$. Thus by part (c) of Theorem 2.1 we can find a complex-valued, even function $\psi_{1} \in L^{2}(\mathbb{R})$ such that $(2 \pi)^{d} f_{0}(t)=\left|\psi_{1}(t)\right|^{2}$ for $t \in \mathbb{R}$ and $\mathcal{F}_{1}^{-1} \psi_{1}(t)=0$ for $|t| \geq \tau / 2$. The idea now is to represent the even function $u_{1}=\mathcal{F}_{1}^{-1} \psi_{1}$ as the Radon transform of a radial function $u$ on $\mathbb{R}^{d}$ that vanishes for $|x| \geq \tau / 2$. The latter is obtained by Radon inversion [50, pp. 22-23], applied separately to the real and imaginary part of the even function $u_{1}$. Then $u_{1}=\mathcal{R}_{d} u$, and the projection theorem implies that $\mathcal{F}_{d} u(x)=\mathcal{F}_{1} \mathcal{R}_{d} u(|x|)=\mathcal{F}_{1} u_{1}(|x|)=\psi_{1}(|x|)$ for $x \in \mathbb{R}^{d}$. Hence

$$
f(x)=f_{0}(|x|)=(2 \pi)^{-d}\left|\psi_{1}(|x|)\right|^{2}=(2 \pi)^{-d}\left|\mathcal{F}_{d} u(x)\right|^{2}, \quad x \in \mathbb{R}^{d},
$$

and the desired convolution representation $\varphi=u \widetilde{*} u$ follows by Fourier inversion. This method of constructing a radial Boas-Kac root is used by Stark and Dimitriadis [59 p. 430] and Janssen [38, p. 334], who suggest that it works in full generality. However, the construction remains valid only if $\psi_{1}$ can be chosen as an even function, i.e., only if conditions (A) and (B) are satisfied. The latter may well fail to hold, and we give explicit counterexamples below.

As in dimension $d=1$ the Boas-Kac root may not be unique, except for the case of a real-valued and radially symmetric Boas-Kac root that is given by (13) up to its sign. In Section 2.3 we used this fact to develop a numerical test for the existence of a real-valued and even Boas-Kac root. Another application of the Radon transform yields an analogous test for the higher-dimensional radial case that uses one-dimensional calculations only. Suppose that $\varphi(x)$ vanishes for $|x| \geq \tau$ and admits a real-valued and radially symmetric Boas-Kac root $u$. Then the Radon transform $u_{1}=\mathcal{R}_{d} u$ is a real-valued and even function with $u_{1}(t)=0$ for $|t| \geq \tau / 2$. Furthermore,

$$
\mathcal{F}_{1} u_{1}(|x|)=\mathcal{F}_{1} \mathcal{R}_{d} u(|x|)=\mathcal{F}_{d} u(x)=(2 \pi)^{d / 2}\left(f_{0}(|x|)\right)^{1 / 2}, \quad x \in \mathbb{R}^{d},
$$

showing that $u_{1}$ can be calculated by one-dimensional Fourier inversion as

$$
u_{1}=(2 \pi)^{d / 2} \mathcal{F}_{1}^{-1} f_{0}^{1 / 2}=(2 \pi)^{-d / 2} \mathcal{F}_{1} f_{0}^{1 / 2}
$$

where $f_{0}^{1 / 2} \in L^{2}(\mathbb{R})$ and hence $u_{1} \in L^{2}(\mathbb{R})$. The test then checks whether $\mathcal{F}_{1} f_{0}^{1 / 2}(t)$ $\neq 0$ for some $t \in \mathbb{R}$ with $|t|>\tau / 2$. If this is true, we conclude that a real-valued and radial Boas-Kac root does not exist. Lemma 2.2 applies with $f_{0}$ in place of $f$.

3.4. Examples. To adapt (18) to the case $d \geq 2$, consider the class of radially symmetric densities $f$ on $\mathbb{R}^{d}$ whose radial part has analytic continuation

$$
f_{0}(z)=c z^{2 p}\left(z^{2}+1\right)^{q}\left(z^{4}+4\right)^{r}\left(\frac{1-\cos z}{z^{2}}\right)^{s} .
$$

Here $p, q$, and $r$ are nonnegative integers, $s$ is a positive integer larger than $p+q+$ $2 r+d / 2$, and the proportionality constant $c$ is chosen such that $f$ is a probability density. Then $f_{0}$ has exponential type $s$ and satisfies condition (A), (B), or (C) if 
and only if $p, q$, or $r$, respectively, is even. All cases in Theorem 3.1 occur within the class, depending on the choice of $p, q, r$, and $d$.

Using polar coordinates, the Fourier transform of a radial function $g$ on $\mathbb{R}^{d}$ can be calculated as

$$
\mathcal{F}_{d} g(x)=\sigma_{d} \int_{0}^{\infty} g_{0}(t) \Omega_{d}(|x| t) t^{d-1} \mathrm{~d} t
$$

where

$$
\Omega_{d}(t)=\Gamma(d / 2)\left(\frac{2}{t}\right)^{(d-2) / 2} J_{(d-2) / 2}(t)
$$

is the radial part of the characteristic function of the uniform probability measure on $\mathbb{S}^{d-1}$ and $J_{(d-2) / 2}$ is a Bessel function. This is repeatedly made use of below.

Example 3.4. The positive definite function $\varphi(x), x \in \mathbb{R}^{3}$, defined by

$$
\varphi(x)= \begin{cases}1-\frac{5}{6}|x|, & |x| \leq 1, \\ \frac{1}{12|x|}\left(15-18|x|+5|x|^{2}\right), & 1 \leq|x| \leq 2, \\ -\frac{1}{12|x|}\left(9-6|x|+|x|^{2}\right), & 2 \leq|x| \leq 3,\end{cases}
$$

and $\varphi(x)=0$ for $|x| \geq 3$ corresponds to the case in which $p=1, q=0, r=$ 0 , and $s=3$ in (31). Condition (A) is violated and a Boas-Kac root does not exist. The density $f$ that is proportional to $(2-\cos |x|)(1-\cos |x|)^{2} /|x|^{4}$ on $\mathbb{R}^{3}$ has a characteristic function of the same form, but with different coefficients. A comparison with Example 2.4 shows that conditions (B) and (C) are violated in this case, so again, a Boas-Kac root does not exist.

The following example presents a radial characteristic function in $\mathbb{R}^{2}$ with a particularly simple nonradial Boas-Kac root.

Example 3.5. The characteristic function $\varphi(x), x \in \mathbb{R}^{2}$, defined by

$$
\varphi(x)=1-\frac{2}{\pi}\left(\arcsin |x|+\left(3|x|-2|x|^{3}\right)\left(1-|x|^{2}\right)^{1 / 2}\right), \quad|x| \leq 1,
$$

and $\varphi(x)=0$ for $|x| \geq 1$ admits a complex-valued nonradial Boas-Kac root $u$, defined by

$$
u(x)=4\left(\frac{2}{\pi}\right)^{1 / 2}\left(x_{1}+i x_{2}\right), \quad|x|=\left(x_{1}^{2}+x_{2}^{2}\right)^{1 / 2} \leq \frac{1}{2},
$$

and $u(x)=0$ otherwise. The radial part $f_{0}$ of the density $f=\mathcal{F}_{2}^{-1} \varphi$ has a zero of order 2 at the origin. Condition (A) is violated and a real-valued and/or radial Boas-Kac root does not exist.

Corollary 3.2 shows that a radial Boas-Kac root exists whenever $\varphi$ is nonnegative. However, a nonnegative $\varphi$ does not necessarily admit a real-valued Boas-Kac root.

Example 3.6. Consider the characteristic function $\varphi(x)=(1-|x|)_{+}^{3}, x \in \mathbb{R}^{3}$. The associated density is

$$
f(x)=\frac{3}{\pi^{2}|x|^{6}}\left(|x| \sin |x|+4 \cos |x|-4+|x|^{2}\right), \quad x \in \mathbb{R}^{3} .
$$


Conditions (A) and (B) hold but it is difficult to check analytically whether (C) is satisfied. The Radon transform argument in Section 3.3 shows that we can apply the numerical test (17) to the radial part $f_{0}$, which is decreasing on the positive half-axis. Putting $x_{1}=1 / 2, x_{2}=11 / 20$, and $A=30$ yields, after renormalizing so that $f_{0}(0)=1$, values of about 0.0117 and 0.0039 for the main term $I$ and error term $E$, respectively. Thus, $\varphi$ does not admit a real-valued Boas-Kac root.

3.5. Factorization of the spherical correlation function. In geostatistics, correlation models are fitted to spatial data [14, and a fundamental requirement on any correlation model is that of positive definiteness. The so-called spherical correlation model, defined by

$$
\varphi(x)=1-\frac{3}{2}|x|+\frac{1}{2}|x|^{3}, \quad x \in \mathbb{R}^{d}, \quad|x| \leq 1,
$$

and $\varphi(x)=0$ for $x \in \mathbb{R}^{d},|x| \geq 1$, is widely used in environmental and geological applications. In geostatistical practice, Euclidean distance $|x|$ in (35) is replaced by $|x| / r$ and the range parameter $r$ is estimated from the data. As shown in 28 . pp. 94-95] the spherical correlation model (35) is a positive definite function in $\mathbb{R}^{d}$ if and only if $d \leq 3$. In what follows, we consider the existence of Boas-Kac roots in dimensions $d=1,2$, and 3 .

If $d=3$, the associated density

$$
f(x)=\mathcal{F}_{3}^{-1} \varphi(x)=\frac{3}{2 \pi^{2}|x|^{6}}\left(4+|x|^{2}-\left(4-|x|^{2}\right) \cos |x|-4|x| \sin |x|\right)
$$

admits the factorization $f(x)=|\psi(x)|^{2}$, where

$$
\psi(x)=\frac{\sqrt{3}}{\pi|x|^{3}}\left(2 \sin \left(\frac{|x|}{2}\right)-|x| \cos \left(\frac{|x|}{2}\right)\right)
$$

is real-valued and radially symmetric. The Boas-Kac root (25) can be computed by Fourier inversion. We recover the well-known result that the spherical correlation function in dimension $d=3$ is the self-convolution of a suitably normalized indicator function of a ball with radius $1 / 2$.

If $d=1$, the density associated with the spherical model is

$$
f(x)=\frac{3}{2 \pi x^{4}}\left(2+x^{2}-2 \cos x-2 x \sin x\right)
$$

which can be factored as $f(x)=|\psi(x)|^{2}$, where

$$
\psi(x)=\left(\frac{3}{2 \pi x^{4}}\right)^{1 / 2}\left(x \sin \left(\frac{x}{2}\right)+i\left(x \cos \left(\frac{x}{2}\right)-2 \sin \left(\frac{x}{2}\right)\right)\right)
$$

is a Hermitian function. Fourier inversion of $\psi$ yields a real-valued Boas-Kac root $u$ given by $u(x)=\sqrt{3} / 2(1-2 x)$ for $|x| \leq 1 / 2$ and $u(x)=0$ for $|x|>1 / 2$, as in Table A.1 of Chilès and Delfiner [14]. By Corollary 3.2 an even Boas-Kac root exists but its explicit form is unknown. A real-valued and even Boas-Kac root does not exist. Indeed, it can be shown as in Section 2 of [12] that the analytic continuation of $f$ has a simple zero near $z=7.461+i 2.089$. Condition $(\mathrm{C})$ is violated and part (d) of Theorem [2.1 applies. Alternatively, the numerical test (17) with $x_{1}=1 / 2$, $x_{2}=11 / 20$, and $A=30$ yields, after renormalizing so that $f(0)=1$ and noting that $f(x)$ decreases for $x>0$, values of about 0.0387 and 0.0131 for the main term $I$ and error term $E$, respectively. 
The case $d=2$ has been an enigma in the geostatistical literature. Oliver [51. p. 944] provides tables of convolution roots and thereby reports that the "square root of the spherical covariance [...] in two dimensions [...] proved to be an intractable problem". Cressie and Pavlicová [18] compute a numerical approximation to a real-valued and symmetric Boas-Kac root, but the resulting convolution does not appear to match the spherical model very closely. A first obstacle toward a rigorous analysis is the calculation of the density $f=\mathcal{F}_{2}^{-1} \varphi$. Table 1 of Mantoglou and Wilson [48] gives a complicated expression for $f$ that attains negative values and is therefore, unfortunately, invalid. The correct hypergeometric expansion

$$
f(x)=\frac{1}{20 \pi}{ }_{1} F_{2}\left(\frac{3}{2} ; 2, \frac{7}{2} ;-\frac{|x|^{2}}{4}\right)
$$

follows from (32), Eq. (6.569) of Gradshteyn and Ryzhik 35], and straightforward manipulation of the coefficients in the resulting power series. As for the existence of Boas-Kac roots, we once more apply Corollary 3.2 A complex-valued, radial Boas-Kac root exists but its explicit form is unknown. A real-valued Boas-Kac root does not exist, which may explain the challenges that Cressie and Pavlicová 18 report. For the renormalized radial part $20 \pi f_{0}, x_{1}=1 / 2, x_{2}=26 / 50$, and $A=30$, the test (17) yields a value of about 0.0173 for the main term $I$, and an upper estimate of about 0.0137 for the error term $E$. Alternatively, surface plots indicate that the radial part $f_{0}(z)$ has a simple zero near $z=8.279+i 1.257$, thereby violating condition $(\mathrm{C})$. We intend to provide details elsewhere.

3.6. Related results. For ease of reference we state, and continually make use of, the following definition.

Definition 3.7. The class $\Phi_{d}^{\tau}$ consists of all continuous, radially symmetric positive definite functions $\varphi(x), x \in \mathbb{R}^{d}$, with $\varphi(0)=1$ and $\varphi(x)=0$ for $|x| \geq \tau$.

Under conditions $(\mathrm{A}),(\mathrm{B})$, and $(\mathrm{C})$, any function $\varphi \in \Phi_{d}^{\tau}$ can be represented as

$$
\varphi=u \tilde{*} u
$$

with a real-valued $u \in L^{2}\left(\mathbb{R}^{d}\right)$ such that $u(x)=0$ for $|x| \geq \tau / 2$; see Theorem 3.1 Assuming only (A) and (B), a complex-valued Boas-Kac root $u=u_{1}+i u_{2}$ exists where both $u_{1} \in L^{2}\left(\mathbb{R}^{d}\right)$ and $u_{2} \in L^{2}\left(\mathbb{R}^{d}\right)$ are real-valued; hence

$$
\varphi=u_{1} \widetilde{*} u_{1}+u_{2} \widetilde{*} u_{2} .
$$

By a result of Rudin [57], all radial, continuous positive definite functions with compact support admit a series representation in analogy to (36) and (37).

Theorem 3.8 (Rudin). For any $\varphi \in \Phi_{d}^{\tau}$ there exists a finite or countably infinite collection of real-valued functions $u_{k} \in L^{2}\left(\mathbb{R}^{d}\right)$ with $u_{k}(x)=0$ for $|x| \geq \tau / 2$ such that

$$
\varphi=\sum_{k} u_{k} \widetilde{*} u_{k}
$$

where the convergence of the sum is uniform.

Rudin's [57] original formulation assumes that $\varphi$ is smooth, and it involves complex-valued functions $u_{k} \in L^{2}\left(\mathbb{R}^{d}\right)$. However, the smoothness assumption in Rudin's Eq. (3) is not required for the representation (38), as is clear from his lemma and straightforward Fourier inversion. Furthermore, we can separate real 
and imaginary parts as in (37) so that each $u_{k}$ is real-valued. However, the $u_{k}$ in (38) are not necessarily radial. A closer look at the arguments in [57] shows that, in fact,

$$
\varphi=\sum_{k} v_{k} \widetilde{*} v_{k}+\sum_{k} \sum_{l=1}^{d} w_{k l} \widetilde{*} w_{k l}
$$

where

$$
w_{k l}(x)=\frac{\partial}{\partial x_{l}} w_{k}(x), \quad x=\left(x_{1}, \ldots, x_{d}\right) \in \mathbb{R}^{d}, \quad l=1, \ldots, d,
$$

with real-valued and radial functions $v_{k} \in L^{2}\left(\mathbb{R}^{d}\right)$ and $w_{k} \in L^{2}\left(\mathbb{R}^{d}\right)$ that vanish for $|x| \geq \tau / 2$, for all $k$.

The following result characterizes the extreme points of the convex set $\Phi_{d}^{\tau}$ defined above. The case $d=1$ is given in Theorem 6 of [63] and Theorem 8 of [64]. For $d \geq 2$, the result is stated in [34] without proof.

Theorem 3.9 (Zastavnyi-Trigub and Gorin). A function $\varphi \in \Phi_{d}^{\tau}$ is an extreme point of $\Phi_{d}^{\tau}$ if and only if the analytic continuation of the radial part $f_{0}$ of its density $f$ is of exact exponential type $\tau$ and has only real zeros.

We omit a proof, since the arguments of Zastavnyi and Trigub 64 for $d=1$ carry over to the case $d \geq 2$ if the terminology and tools of Sections 3.1 and 3.2 are used. Alternative proofs of the result for $d \geq 2$ use Rudin's representation (39) and the Radon transform methods of Section 3.3. Perhaps surprisingly, there are extreme points of the class $\Phi_{d}^{\tau}$ that do not admit a real-valued Boas-Kac root. If $d \geq 3$, there exist extreme points that do not admit any Boas-Kac root. These cases occur whenever $f_{0}$ is of exact exponential type $\tau$, and $f$ has only real zeros, and violates condition (A). For instance, the characteristic function (34) is an extreme point of the class $\Phi_{3}^{3}$ that does not admit a Boas-Kac root. In terms of Rudin's series representation (39), a detailed study of the arguments in [57, pp. 51-52] shows that an extreme point $\varphi$ that does not admit a real-valued Boas-Kac root can be expressed as

$$
\varphi=\sum_{l=1}^{d} w_{1 l} \widetilde{*} w_{1 l},
$$

with all the $v_{k}$ terms and all but one of the $w_{k}$ terms in the series vanishing.

\section{TURÁN's PROBLEM}

4.1. Boas-Kac bounds, the Lukosz staircase, and Turán's problem. Boas and $\mathrm{Kac}\left[9\right.$ showed that if $\varphi \in \Phi_{1}^{\tau}$, i.e., $\varphi$ is a continuous, even positive definite function on $\mathbb{R}$ with $\varphi(0)=1$ and $\varphi(x)=0$ for $|x| \geq \tau$, then one has

$$
|\varphi(\tau x)| \leq \cos \frac{\pi}{\lceil 1 /|x|\rceil+1}, \quad|x|<1,
$$

where $\lceil y\rceil$ denotes the least integer larger than or equal to $y$. Furthermore, the estimate is sharp. This result has various applications and has been rediscovered both in the optics [46, 47] and in the statistics literature [13, 19]. In optics the graph of the upper bound in (41) has become known as the Lukosz staircase. Under the same assumptions, Boas and Kac [9] also proved the integral bound

$$
\int|\varphi(x)| \mathrm{d} x \leq \tau
$$


Similar to the development of (41), this inequality has been rediscovered and applied to problems in optics [46, 47] and time series analysis [3]. More recently, questions of this type have attracted considerable attention in the mathematical literature, where they are referred to as Turán's problem, and discussed in the works of Gorbachev [33, Arestov, Berdysheva, and Berens [4, 5, 6, and Kolountzakis and Révész 41 42, among others.

In what follows we give analogues and generalizations of the Boas-Kac bounds to compactly supported positive definite functions in $\mathbb{R}^{d}, d \geq 2$. The main result in Section 4.2 is the pointwise bound (43). Its proof relies on Rudin's convolution representation (38) and is deferred to Section 4.3. In the brief Section 4.4 we review Gorbachev's recent extension of the integral bound (42).

4.2. Pointwise bounds for radial functions. Our key result here is a pointwise bound for radial positive definite functions defined on $\mathbb{R}^{d}, d \geq 2$. The result for $d=2$ is stated in Section 3.1 of [39], with a proof that depends on the existence of a Boas-Kac root and is therefore incomplete.

Theorem 4.1. Let $d \geq 2$. For any $\varphi \in \Phi_{d}^{\tau}$ one has

$$
|\varphi(\tau x)| \leq G_{d}(\kappa(|x|)), \quad|x| \in[1 / 2,1),
$$

where

$$
G_{d}(t)=\frac{\Gamma\left(\frac{d}{2}\right)}{\Gamma\left(\frac{1}{2}\right) \Gamma\left(\frac{d-1}{2}\right)} \int_{t}^{1}\left(1-s^{2}\right)^{(d-3) / 2} \mathrm{~d} s, \quad t \in[0,1],
$$

and $\kappa(t)$ is defined piecewise as $\kappa(t)=\left(1-\left(4 t^{2}\right)^{-1}\right)^{1 / 2}$ if $t \in[1 / 2, \sqrt{2} / 2)$ and $\kappa(t)=t$ if $t \in[\sqrt{2} / 2,1)$.

Applications are straightforward. Consider the truncated power function $\varphi(x)=$ $\left(1-|x|^{\lambda}\right)_{+}^{\kappa}, x \in \mathbb{R}^{d}$. Kuttner [44] and Golubov 32] showed that for $\lambda \in(0,2)$ and $d=1,2, \ldots$ the truncated power function is positive definite if and only if $\kappa \geq k_{d}(\lambda)$ where the right-hand side is a nondecreasing function of $\lambda$. The lower bounds on $k_{d}(\lambda)$ that follow from (43) complement recent results in [29] and [31.

The estimate (43) applies to nonradial functions if we replace the point value $\varphi(x)$ by the spherical average at $|x| \in[1 / 2,1)$. Indeed, let $H$ denote normalized Haar measure on the group $S O(d)$ of rotations $\rho$ in $\mathbb{R}^{d}$. Given a continuous positive definite function $\varphi(x), x \in \mathbb{R}^{d}$, with $\varphi(0)=1$ and $\varphi(x)=0$ for $|x| \geq 1$, its radialization

$$
\varphi_{\text {rad }}(x)=\int \varphi(\rho x) H(\mathrm{~d} \rho)
$$

is a radially symmetric function for which the assumptions of Theorem 4.1 hold. Among nonradial positive definite functions, those depending on an $l_{\alpha}$ norm or quasinorm have been of particular interest. We refer to [62] and Section 4 of [29] for recent results and reviews, and to Lemma 3.7 of [27] for a pointwise bound on $\alpha$-symmetric positive definite functions with compact support.

For $d=1,2, \ldots$ and $t \in(0,1)$ define

$$
m_{d}(t)=\inf _{\varphi \in \Phi_{d}^{1}} \varphi_{0}(t) \quad \text { and } \quad M_{d}(t)=\sup _{\varphi \in \Phi_{d}^{1}} \varphi_{0}(t) .
$$



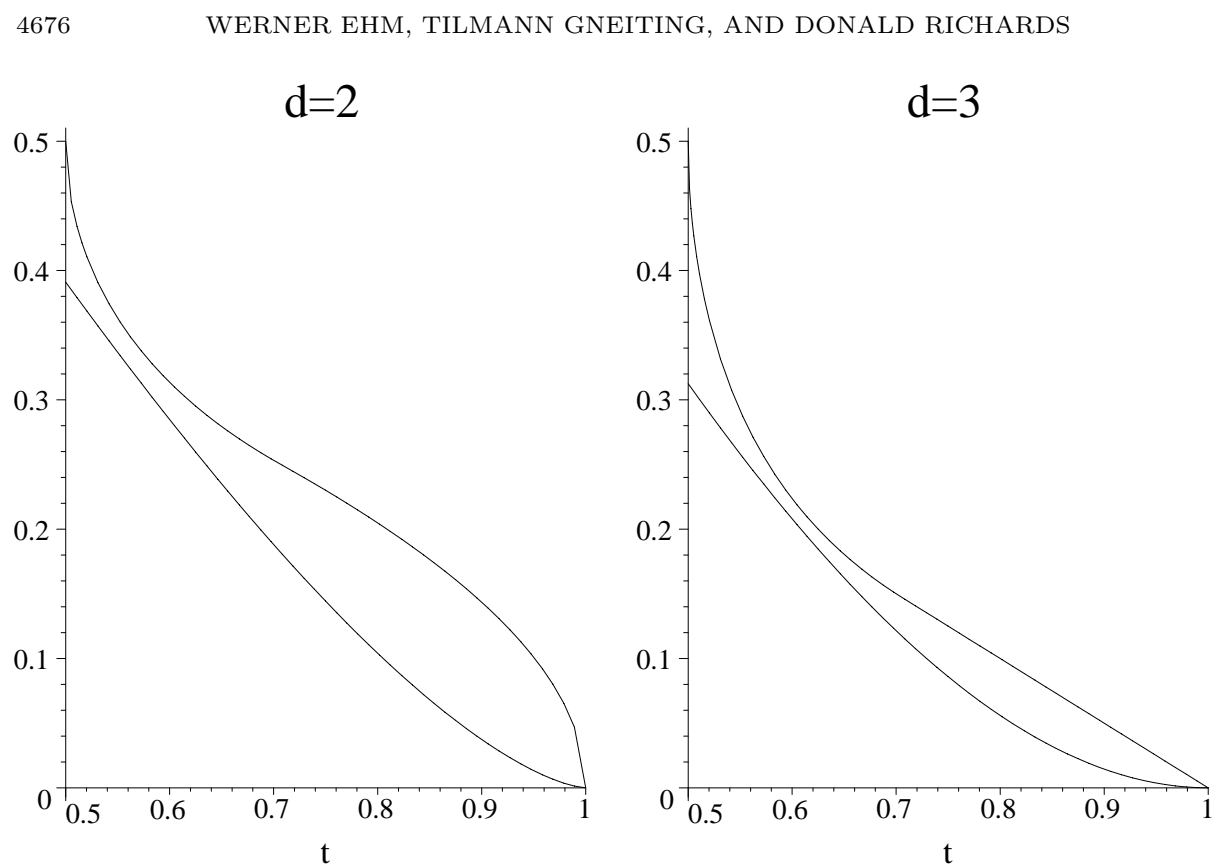

Figure 2. The lower estimate (45) and the upper estimate 46] of $M_{d}(t)$, for $d=2$ (left) and $d=3$ (right).

Boas and Kac [9] showed that both $-m_{1}(t)$ and $M_{1}(t)$ equal the upper bound in (41). If $d \geq 2$, only partial results are available. The Euclid's hat function

$$
\varphi(x)=2 \frac{\Gamma\left(\frac{d+2}{2}\right)}{\Gamma\left(\frac{1}{2}\right) \Gamma\left(\frac{d+1}{2}\right)} \int_{|x|}^{1}\left(1-s^{2}\right)_{+}^{(d-1) / 2} \mathrm{~d} s, \quad x \in \mathbb{R}^{d},
$$

is the self-convolution of a suitably normalized indicator function of a $d$-dimensional ball of radius $1 / 2$ and therefore belongs to the class $\Phi_{d}^{1}$. Using the notation of Theorem 4.1 we find the lower estimate

$$
M_{d}(t) \geq 2 G_{d+2}(t), \quad t \in(0,1),
$$

while (43) implies the upper estimate

$$
M_{d}(t) \leq G_{d}(\kappa(t)), \quad t \in[1 / 2,1),
$$

and an analogous estimate for $m_{d}(t)$. As Figures 2 and 3 illustrate, the estimates (45) and (46) are surprisingly sharp. Finally,

$$
\inf _{r>0} \Omega_{d}(r) \leq m_{d}(t) \leq M_{d}(t) \inf _{r>0} \Omega_{d}(r), \quad t \in(0,1),
$$

where $\Omega_{d}$ is defined by (33). The first inequality in (47) is immediate from Schoenberg's representation 58 of a radial characteristic function as a mixture of the functions $\Omega_{d}(r|x|), x \in \mathbb{R}^{d}$, over $r>0$, that is, the representation (32) with $g$ being a probability density. If $\varphi$ belongs to the class $\Phi_{d}^{1}$, then so does the product $\varphi(x) \Omega_{d}(r|x|)$ for all $r>0$, thereby proving the second inequality. Frieden [23] provides numerical estimates of $M_{2}(t)$ while Barakat and Newsam [7] give approximations to both $m_{2}(t)$ and $M_{2}(t)$. The numerical results in the latter reference violate the second inequality in (47) in various instances and are therefore inconsistent. 

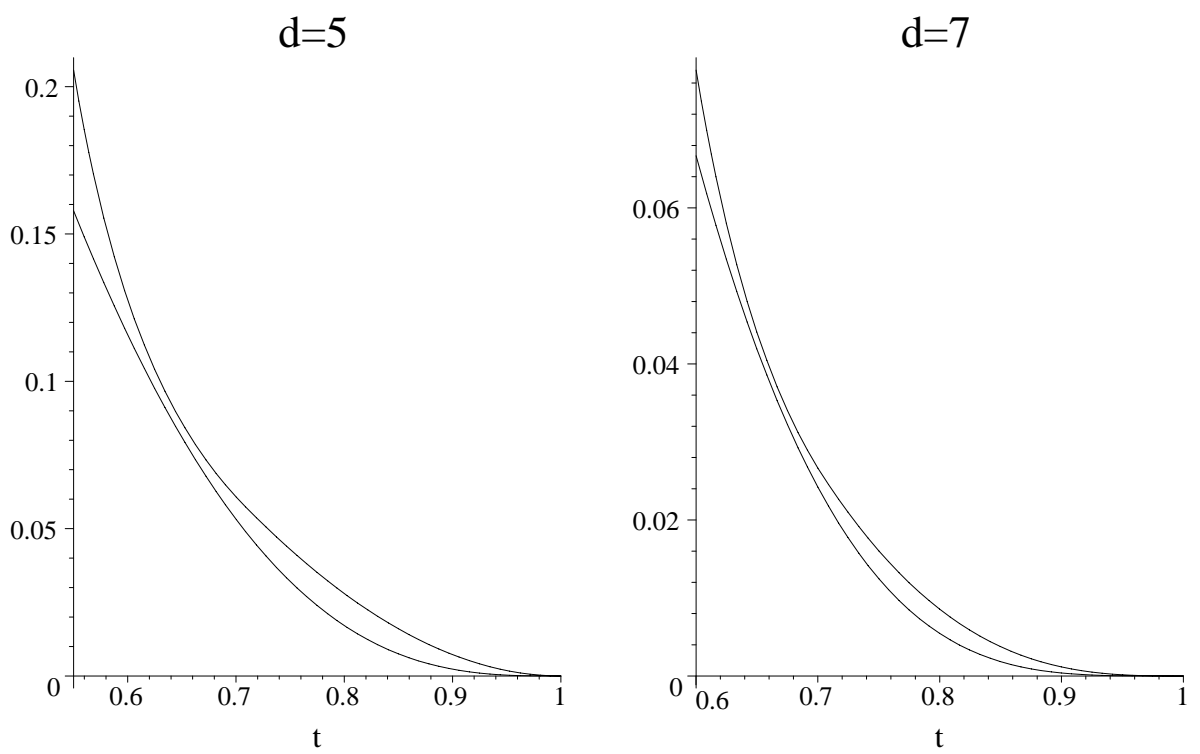

Figure 3. The lower estimate (45) and the upper estimate (46) of $M_{d}(t)$, for $d=5$ (left) and $d=7$ (right).

4.3. Proof of Theorem 4.1 Without loss of generality, we may assume that $\tau=1$. Let $H$ denote normalized Haar measure on the group $S O(d)$ of rotations $\rho$ in $\mathbb{R}^{d}$, and consider Rudin's representation (38) of a function $\varphi \in \Phi_{d}^{1}$. For each $k$ the function

$$
h_{k}(y)=\int\left|u_{k}(\rho y)\right|^{2} H(\mathrm{~d} \rho), \quad y \in \mathbb{R}^{d},
$$

is radially symmetric and such that

$$
\int h_{k}(y) \mathrm{d} y=\int\left|u_{k}(y)\right|^{2} \mathrm{~d} y=\left(u_{k} \widetilde{*} u_{k}\right)(0) .
$$

The latter terms sum to $\varphi(0)=1$. Hence

$$
\begin{aligned}
& |\varphi(x)|=\int|\varphi(\rho x)| H(\mathrm{~d} \rho) \leq \sum_{k} \int\left|\left(u_{k} \widetilde{*} u_{k}\right)(\rho x)\right| H(\mathrm{~d} \rho) \\
& \leq \sum_{k} \int_{A_{x}} h_{k}(y) \mathrm{d} y \leq G_{d}(\kappa(|x|)) \sum_{k} \int h_{k}(y) \mathrm{d} y=G_{d}(\kappa(|x|))
\end{aligned}
$$

where the second and third inequalities are justified by (48) and (49), respectively. Thus, the two lemmas below complete the proof of the theorem.

Lemma 4.2. Suppose that $v \in L^{2}\left(\mathbb{R}^{d}\right)$ vanishes for $|x| \geq 1 / 2$. Let $x \in \mathbb{R}^{d}$ with $|x| \in[1 / 2,1]$ and put $A_{x}=\left\{y \in \mathbb{R}^{d}:|y| \leq 1 / 2,|y+x| \leq 1 / 2\right\}$. Then

$$
\int|(v \widetilde{*} v)(\rho x)| H(\mathrm{~d} \rho) \leq \int_{A_{x}} \int|v(\rho y)|^{2} H(\mathrm{~d} \rho) \mathrm{d} y .
$$


Proof. Note that $|v(y)||v(y+\rho x)|$ vanishes unless $y \in A_{\rho x}$. Hence

$$
\begin{aligned}
& \int|(v \widetilde{*} v)(\rho x)| H(\mathrm{~d} \rho) \leq \iint|v(y)||v(y+\rho x)| \mathrm{d} y H(\mathrm{~d} \rho) \\
& \quad \leq\left(\iint_{A_{\rho x}}|v(y)|^{2} \mathrm{~d} y H(\mathrm{~d} \rho) \iint_{A_{\rho x}}|v(y+\rho x)|^{2} \mathrm{~d} y H(\mathrm{~d} \rho)\right)^{1 / 2}
\end{aligned}
$$

and it suffices to show that either double integral under the square root equals the right-hand side of (48). Consider the second integral. Within the inner integral $\rho$ is fixed. Under the change of variables $y \rightarrow z=\rho^{-1}(y+\rho x)$ the integration region $y \in A_{\rho x}$ is mapped to $z \in A_{-x}=-A_{x}$ which does not depend on $\rho$. By Fubini's theorem and radial symmetry,

$$
\begin{aligned}
\iint_{A_{\rho x}}|v(y+\rho x)|^{2} \mathrm{~d} y H(\mathrm{~d} \rho)=\iint_{-A_{x}}|v(\rho z)|^{2} \mathrm{~d} z H(\mathrm{~d} \rho) \\
=\int_{-A_{x}} \int|v(\rho z)|^{2} H(\mathrm{~d} \rho) \mathrm{d} z=\int_{A_{x}} \int|v(\rho z)|^{2} H(\mathrm{~d} \rho) \mathrm{d} z .
\end{aligned}
$$

The first integral under the root is handled similarly, by substituting $y=\rho z$.

Lemma 4.3. Suppose that $h \in L^{1}\left(\mathbb{R}^{d}\right)$ is nonnegative and radial. If $x$ and $A_{x}$ are as in Lemma 4.2, then

$$
\int_{A_{x}} h(y) \mathrm{d} y \leq G_{d}(\kappa(|x|)) \int h(y) \mathrm{d} y .
$$

Proof. Fix $x \in \mathbb{R}^{d}$ with $|x| \in[1 / 2,1]$ and let $K_{x}$ denote the smallest spherical cone

$$
K_{x, \alpha}=\left\{y \in \mathbb{R}^{d}: \frac{y \cdot x}{|y||x|} \geq \cos \alpha\right\}
$$

that contains the set $A_{-x}$. Geometric arguments in analogy to those in Section 3.1 of Janssen [39] show that the smallest admissible angle $\alpha$ satisfies the relation $\cos \alpha=\kappa(|x|)$. Let $\mu$ denote normalized uniform measure on the unit sphere $\mathbb{S}^{d-1}$ and let $K_{x}^{1}=K_{x} \cap \mathbb{S}^{d-1}$. Then

$$
\begin{aligned}
\int_{A_{x}} h(y) \mathrm{d} y & =\int_{A_{-x}} h(y) \mathrm{d} y \leq \int_{K_{x}} h(y) \mathrm{d} y \\
& =\sigma_{d} \int_{\theta \in K_{x}^{1}} \int_{r=0}^{\infty} h_{0}(r) r^{d-1} \mathrm{~d} r \mu(\mathrm{d} \theta)=\mu\left(K_{x}^{1}\right) \int h(y) \mathrm{d} y .
\end{aligned}
$$

If $\theta$ is a random vector with distribution $\mu$, then for every $\xi \in \mathbb{S}^{d-1}$ the projection length $\lambda=\theta \cdot \xi$ is a random variable that takes values in $[-1,1]$ and whose density $g_{d}(\lambda)$ is proportional to $\left(1-\lambda^{2}\right)^{(d-3) / 2}$. Hence

$$
\mu\left(K_{x}^{1}\right)=\int_{\kappa(|x|)}^{1} g_{d}(s) \mathrm{d} s=G_{d}(\kappa(|x|))
$$

and the proof of the estimate (49) is complete. 
4.4. Integral bounds. In this section we review Gorbachev's 33 multivariate analogue of the integral bound (42). Note that $\varphi$ is not assumed to be radial.

Theorem 4.4 (Gorbachev). Suppose that $\varphi(x), x \in \mathbb{R}^{d}$, is a continuous positive definite function with $\varphi(0)=1$ and $\varphi(x)=0$ for $|x| \geq \tau$. Then

$$
\int \varphi(x) \mathrm{d} x \leq 2^{-d} \tau^{d} v_{d}
$$

where $v_{d}=\pi^{d / 2} / \Gamma\left(\frac{d}{2}+1\right)$ denotes the volume of the unit ball in $\mathbb{R}^{d}$.

The elegant recent proof of Kolountzakis and Révész [41] uses the radialization formula (44) and Rudin's series representation (38) to show that

$$
\begin{aligned}
& \int \varphi(x) \mathrm{d} x=\int \varphi_{\mathrm{rad}}(x) \mathrm{d} x \leq \sum_{k} \iint\left|u_{k}(y)\right|\left|u_{k}(y+x)\right| \mathrm{d} y \mathrm{~d} x \\
& =\sum_{k}\left(\int\left|u_{k}(y)\right| \mathrm{d} y\right)^{2} \leq 2^{-d} \tau^{d} v_{d} \sum_{k} \int\left|u_{k}(y)\right|^{2} \mathrm{~d} y=2^{-d} \tau^{d} v_{d}
\end{aligned}
$$

by the fact that each $u_{k}$ is supported on a ball of radius $\tau / 2$, the Cauchy-Schwarz inequality, and the relationship $\sum_{k} \int\left|u_{k}(y)\right|^{2} \mathrm{~d} y=\varphi(0)=1$. For radial functions, the first equality in (51) remains valid with $\varphi$ and $\varphi_{\text {rad }}$ replaced by their absolute values, respectively, and we obtain a slightly stronger result.

Corollary 4.5. If $\varphi \in \Phi_{d}^{\tau}$, then

$$
\int|\varphi(x)| \mathrm{d} x \leq 2^{-d} \tau^{d} v_{d}
$$

If $d=2$ the corollary reduces to Proposition 7.2 of [38. In Eq. $(3.2,7 \mathrm{a})$ of [47, the estimate (52) is stated for $d=2$ and general, not necessarily radial functions. However, the proofs in these references depend on the existence of a Boas-Kac root and therefore are incomplete. We conjecture, but have not been able to prove, that (52) remains valid under the conditions of Theorem 4.4 .

Gorbachev's bound (50) solves a specific instance of Turán's integral problem: Given a closed convex centrally symmetric domain $D$ in $\mathbb{R}^{d}$, maximize $\int \varphi(x) \mathrm{d} x$ over the class of continuous positive definite functions $\varphi(x), x \in \mathbb{R}^{d}$, with $\varphi(0)=1$ and support contained in D. Theorem 4.4 shows that the Euclidean ball is a Turán domain, meaning that the maximum is achieved by the self-convolution of a properly rescaled indicator function of the half-body. Kolountzakis and Révész 41] and Arestov and Berdysheva [5] show that a convex closed centrally symmetric polytope in $\mathbb{R}^{d}$ is a Turán domain if it admits a tiling by translation. It is not presently known whether non-Turán domains exist.

\section{Minimum VARiance THEOREM}

5.1. Extremal problems for characteristic functions and densities. This section provides a unified approach to a class of previously scattered extremal problems that arise in various formulations and various applications, ranging from numerical Fourier inversion [11], to spectral density estimation [54, 40, imaging [53, 59, and spatial statistics [30. Let $\Delta=\sum_{l=1}^{d} \partial^{2} / \partial x_{l}^{2}$ denote the Laplace operator on $\mathbb{R}^{d}$. We consider the following optimization problem: Minimize $-\Delta \varphi(0)$ over the class of characteristic functions $\varphi$ on $\mathbb{R}^{d}$, subject to the condition that $\varphi(x)=0$ for $|x| \geq \tau$. In terms of probability densities, the problem is equivalent 
to a minimum variance problem: Minimize $\int|x|^{2} f(x) \mathrm{d} x$, subject to the condition that $\mathcal{F}_{d} f(x)=0$ for $|x| \geq \tau$. Indeed, if the random vector $X=\left(X_{1}, \ldots, X_{d}\right)$ has characteristic function $\varphi=\mathcal{F}_{d} f$, then

$$
\int|x|^{2} f(x) \mathrm{d} x=E|X|^{2}=E\left(X_{1}^{2}+\cdots+X_{d}^{2}\right)=-\Delta \varphi(0) .
$$

In particular, $\varphi$ is twice differentiable if and only if $f$ has a finite second moment. If this is not the case, we put $-\Delta \varphi(0)=+\infty$. Note that radial symmetry is not assumed here.

The following result is stated for $\tau=1$ only, as the analogue for $\tau>0$ is obvious. Recall the definition of $\Omega_{d}$ in (33) and put

$$
\gamma_{d}^{2}=\frac{4 j_{(d-2) / 2}^{d-2}}{\pi^{d / 2} \Gamma(d / 2) J_{d / 2}^{2}\left(j_{(d-2) / 2}\right)} \quad \text { and } \quad c_{d}=\frac{4 j_{(d-2) / 2}^{2}}{4^{d} \pi^{d / 2} \Gamma(d / 2)}
$$

for $d=1,2, \ldots$, where $j_{\nu}$ denotes the first positive zero of the Bessel function $J_{\nu}$.

Theorem 5.1. Let $\varphi$ be the characteristic function of a probability density $f$ on $\mathbb{R}^{d}$ and suppose that $\varphi(x)=0$ for $|x| \geq 1$. Then

$$
-\Delta \varphi(0)=\int|x|^{2} f(x) \mathrm{d} x \geq 4 j_{(d-2) / 2}^{2},
$$

with equality if and only if $\varphi=\omega_{d} \widetilde{*} \omega_{d}$, where $\omega_{d}(x)=\gamma_{d} \Omega_{d}\left(2 j_{(d-2) / 2}|x|\right)$ for $|x| \leq 1 / 2$ and $\omega_{d}(x)=0$ for $|x| \geq 1 / 2$. The corresponding minimum variance density is

$$
f(x)=c_{d}\left(\frac{\Omega_{d}(|x| / 2)}{j_{(d-2) / 2}^{2}-(|x| / 2)^{2}}\right)^{2} .
$$

If $\varphi$ is radial, the estimate (54) can be reformulated as

$$
-\varphi_{0}^{\prime \prime}(0) \geq \frac{4}{d} j_{(d-2) / 2}^{2}
$$

Theorem 5.1 implies that the right-hand side of $(56)$ is a strictly increasing function of $d$, and we note in passing that this increasing property implies

$$
j_{n / 2}>\left(\frac{n+2}{n+1}\right)^{1 / 2} j_{(n-1) / 2}, \quad n=0,1,2, \ldots
$$

which forms a special case of Theorem 2 in Ismail and Muldoon [37. For the case in which $d=1$, the theorem is due to Bohman 11] and Papoulis [54]. The minimizing characteristic function then is

$$
\varphi(x)=(1-|x|) \cos (\pi x)+\frac{1}{\pi} \sin (\pi|x|), \quad x \in \mathbb{R}, \quad|x| \leq 1,
$$

and (56) reads $-\varphi_{0}^{\prime \prime}(0) \geq \pi^{2}$. Note that in this case the inequality for $-\varphi_{0}^{\prime \prime}(0)$ can also be obtained from (41). The case $d=2$ has been considered by Papoulis [53] and Stark and Dimitriadis [59]. The results in these papers assume radial symmetry as well as the existence of a Boas-Kac root. As Theorem 3.1 shows, a Boas-Kac representation may fail to exist if $d \geq 2$, and our proof uses Rudin's series representation (38) instead. The case $d=3$ recovers a result of Kanter [40] in the form given in [30]. The minimizing characteristic function is

$$
\varphi(x)=(1-|x|) \frac{\sin (2 \pi|x|)}{2 \pi|x|}+\frac{1}{\pi} \frac{1-\cos (2 \pi|x|)}{2 \pi|x|}, \quad x \in \mathbb{R}^{3}, \quad|x| \leq 1,
$$


and (56) reduces to $-\varphi_{0}^{\prime \prime}(0) \geq 4 / 3 \pi^{2}$. The statements in dimension $d \geq 4$, the general result in dimension $d=2$, and our unified approach are new. Note the similarity of the minimizing density (55) to the solution of the extremal problem in Section 3 of [15] and Section 6 of [16], with its interesting and surprising connections to the sphere packing problem.

The following minimum variance result for unimodal, radially symmetric densities is due to Kanter [40] if $d=1$. The general case is new. Theorem 5.1 is proved in Section 5.2, and the proof of Corollary 5.2 is deferred to Section 5.3.

Corollary 5.2. Suppose that $f$ is a unimodal, radially symmetric probability density on $\mathbb{R}^{d}$ whose characteristic function $\varphi(x)$ vanishes for $|x| \geq 1$. Then

$$
\int|x|^{2} f(x) \mathrm{d} x \geq \frac{4 d}{d+2} j_{d / 2}^{2}
$$

with equality if and only if

$$
f(x)=2 \pi \int_{|x|}^{\infty} g_{0}(t) t \mathrm{~d} t
$$

where $g_{0}$ is the radial part of the minimum variance density for dimension $d+2$ in Theorem 5.1 .

5.2. Proof of Theorem 5.1. Since (53) is invariant under radialization, we may assume that $\varphi$ and $f$ are radial functions. By Theorem 3.8 there exist real-valued functions $u_{k} \in L^{2}\left(\mathbb{R}^{d}\right)$ vanishing for $|x| \geq 1 / 2$ such that

$$
f=(2 \pi)^{-d} \mathcal{F}_{d} \varphi=(2 \pi)^{-d} \sum_{k}\left|\mathcal{F}_{d} u_{k}\right|^{2} .
$$

By Parseval's formula,

$$
\frac{1}{(2 \pi)^{d}} \int\left|-i x_{l} \mathcal{F}_{d} u_{k}(x)\right|^{2} \mathrm{~d} x=\int\left|\frac{\partial}{\partial x_{l}} u_{k}(x)\right|^{2} \mathrm{~d} x
$$

for $k \geq 1$ and $l=1, \ldots, d$, which upon summing gives

$$
\int|x|^{2} f(x) \mathrm{d} x=\frac{1}{(2 \pi)^{d}} \sum_{k} \sum_{l} \int\left|x_{l} \mathcal{F}_{d} u_{k}(x)\right|^{2} \mathrm{~d} x=\sum_{k} \int\left|\nabla u_{k}(x)\right|^{2} \mathrm{~d} x .
$$

This allows us to draw on the variational formulation of the eigenvalue problem for the Laplace operator with Dirichlet boundary conditions on the ball $B$ of radius $1 / 2$ centered at the origin of $\mathbb{R}^{d}$. The minimum of the Rayleigh quotient gives the smallest eigenvalue $\lambda_{d}$ of the problem $-\Delta u=\lambda u$ in $B, u=0$ on $\partial B$, i.e.,

$$
\frac{\int_{B}|\nabla u|^{2}}{\int_{B}|u|^{2}} \geq \lambda_{d}
$$

with equality if and only if $u$ is proportional to $v_{d}$, the associated first eigenfunction. See, e.g., Section IV.2 in [17]. Inserting (60) into (59) and observing that $\sum_{k} \int\left|u_{k}\right|^{2}=\varphi(0)=1$, we obtain

$$
\int|x|^{2} f=\sum_{k} \frac{\int\left|\nabla u_{k}\right|^{2}}{\int\left|u_{k}\right|^{2}} \int\left|u_{k}\right|^{2} \geq \lambda_{d} \sum_{k} \int\left|u_{k}\right|^{2}=\lambda_{d}
$$

with equality if and only if the sum reduces to a single term $u_{k}=u$ that is proportional to $v_{d}$. By (58), the optimal density $f$ is proportional to $\left|\mathcal{F}_{d} v_{d}\right|^{2}$, and the optimal characteristic function $\varphi$ is proportional to $v_{d} \widetilde{*} v_{d}$. It remains to determine 
the relevant functions and constants. The well-known expressions for $v_{d}$ and $\lambda_{d}$ are obtained as a by-product of the following calculations.

Let $\mu$ denote the normalized uniform distribution on $\mathbb{S}^{d-1}$. The definition of $\omega_{d}$ and the fact that $\Omega_{d}(|x|)=\int e^{i x \cdot y} \mu(\mathrm{d} y)$ for $x \in \mathbb{R}^{d}$ show that $\omega_{d}$ is an eigenfunction of $-\Delta$, with associated eigenvalue $\left(2 j_{(d-2) / 2}\right)^{2}$ and vanishing on the boundary $\partial B$ of $B$. Since $\omega_{d}$ is positive within $B$, it is the unique first eigenfunction corresponding to the minimal eigenvalue, and we conclude that $v_{d}$ is proportional to $\omega_{d}$, and $\lambda_{d}=4 j_{(d-2) / 2}^{2}$. The factor $\gamma_{d}$ is obtained from Eq. (6.521.1) in Gradshteyn and Ryzhik [35. As for the calculation of the corresponding density $f$, Green's formula gives

$$
\begin{aligned}
& 4 j_{(d-2) / 2}^{2} \mathcal{F}_{d} \omega_{d}(x)=\int_{B} e^{i x \cdot y}\left(4 j_{(d-2) / 2}^{2}\right) \omega_{d}(y) \mathrm{d} y=-\int_{B} e^{i x \cdot y} \Delta \omega_{d}(y) \mathrm{d} y \\
& \quad=-\int_{B}\left(\Delta e^{i x \cdot y}\right) \omega_{d}(y) \mathrm{d} y+\int_{\partial B}\left(\partial_{n} e^{i x \cdot y}\right) \omega_{d}(y) \mathrm{d} o-\int_{\partial B} e^{i x \cdot y} \partial_{n} \omega_{d}(y) \mathrm{d} o
\end{aligned}
$$

where do denotes integration with respect to surface measure on $\partial B$, and $\partial_{n}$ is the derivative in the direction of the outward pointing normal vector. By radial symmetry, $\partial_{n} \omega_{d}(y)$ is constant on $\partial B$. Explicitly, $\partial_{n} \omega_{d}(y)=2 j_{(d-2) / 2} \Omega_{d}^{\prime}\left(j_{(d-2) / 2}\right) \gamma_{d}=$ $\beta_{d}$, say, hence

$$
\int_{\partial B} e^{i x \cdot y} \partial_{n} \omega_{d}(y) \mathrm{d} o=2^{-(d-1)} \sigma_{d} \beta_{d} \int e^{i x \cdot(y / 2)} \mu(\mathrm{d} y)=2^{-d+1} \sigma_{d} \beta_{d} \Omega_{d}(|x| / 2) .
$$

The other surface integral equals zero because $\omega_{d}$ vanishes on the boundary of $B$. Finally, $-\int_{B}\left(\Delta e^{i x \cdot y}\right) \omega_{d}(y) \mathrm{d} y=|x|^{2} \mathcal{F}_{d} \omega_{d}(x)$, so we get from (61)

$$
\mathcal{F}_{d} \omega_{d}(x)=2^{-d-1} \sigma_{d} \beta_{d} \frac{\Omega_{d}(|x| / 2)}{j_{(d-2) / 2}^{2}-(|x| / 2)^{2}} .
$$

The constant factor is easily evaluated, and since $f=(2 \pi)^{-d}\left|\mathcal{F}_{d} \omega_{d}\right|^{2}$ the proof of Theorem 5.1 is complete.

5.3. Proof of Corollary [5.2, By Proposition 1 of Eaton [21] or Eq. (36) in [27, the density $f$ of a radial characteristic function $\varphi(x)=\varphi_{0}(|x|), x \in \mathbb{R}^{d}$, is unimodal if and only if $\varphi_{0}(|x|), x \in \mathbb{R}^{d+2}$, is a characteristic function. Hence

$$
\int|x|^{2} f(x) \mathrm{d} x=-\Delta \varphi(0)=-d \varphi_{0}^{\prime \prime}(0) \geq \frac{4 d}{d+2} j_{d / 2}^{2},
$$

with equality if and only if $\varphi_{0}$ is the radial part of the extremal characteristic function for dimension $d+2$ in Theorem 5.1. The associated minimizing density is the $d$-dimensional marginal density of the minimum variance density for dimension $d+2$ in Theorem 5.1] The proof of the corollary is complete.

\section{ACKNOWLEDGEMENTS}

We are grateful to Richard Askey, Michael Baake, Elena Berdysheva, Piotr Graczyk, Enrico Laeng, Jean-Jacques Loeb, Carlo Morpurgo, Martin Muldoon, Christian Simader, Boris Solomyak, Victor Zastavnyi, and a particularly helpful anonymous reviewer for comments, discussions, and references. Tilmann Gneiting acknowledges support by the National Science Foundation through a Career Award, Award No. 0134264, and by the DoD Multidisciplinary University Research Initiative (MURI) program administered by the Office of Naval Research under Grant No. N00014-01-10745. 


\section{REFERENCES}

1. N. I. Achieser, Theory of approximation, Frederick Ungar, New York, 1956. MR 20:1872

2. E. J. Akutowicz, On the determination of the phase of a Fourier integral, II, Proc. Amer. Math. Soc. 8 (1957), 234-238. MR 18:895c

3. O. D. Anderson, Bounding sums for the autocorrelations of moving average processes, Biometrika 62 (1975), 706-707. MR 52:12265

4. V. V. Arestov and E. E. Berdysheva, Turán's problem for positive definite functions with support in a hexagon, Proc. Steklov Math. Inst., Suppl., 2001, pp. S20-S29.

5. - The Turán problem for a class of polytopes, East J. Approx. 8 (2002), 381-388. MR 2003i: 42010

6. V. V. Arestov, E. E. Berdysheva, and H. Berens, On pointwise Turán's problem for positive definite functions, East J. Approx. 9 (2003), 31-42. MR 2004b:42014

7. R. Barakat and G. Newsam, Upper and lower bounds on radially symmetric optical transfer functions, Optica Acta 29 (1982), 1191-1204.

8. R. P. Boas, Entire functions, Academic Press, New York, 1954. MR 16:914f

9. R. P. Boas and M. Kac, Inequalities for Fourier transforms of positive functions, Duke Math. J. 12 (1945), 189-206, Errata 15 (1948), 107-109. MR 6:265h

10. S. Bochner, Monotone Funktionen, Stieltjessche Integrale und harmonische Analyse, Math. Ann. 108 (1933), 378-410.

11. H. Bohman, Approximate Fourier analysis of distribution functions, Ark. Mat. 4 (1960), 99-157. MR 23:A3963

12. H. Carnal and M. Dozzi, On a decomposition problem for multivariate probability measures, J. Multivariate Anal. 31 (1989), 165-177. MR 91c:60016

13. K. C. Chanda, On bounds of serial correlations, Ann. Math. Statist. 33 (1962), 1457-1460. MR 26:872

14. J.-P. Chilès and P. Delfiner, Geostatistics, Wiley, New York, 1999. MR 2000f:86010

15. H. Cohn, New upper bounds of sphere packings. II, Geom. Topol. 6 (2002), 329-353. MR 2004b:52032

16. H. Cohn and N. Elkies, New upper bounds of sphere packings I, Ann. of Math. 157 (2003), 689-714. MR 2004b:11096

17. R. Courant and D. Hilbert, Methods of mathematical physics, vol. 1, Interscience Publishers, New York, 1953. MR 16:426a

18. N. Cressie and M. Pavlicová, Calibrated spatial moving average simulations, Stat. Model. 2 (2002), 1-13.

19. N. Davies, M. B. Pate, and M. G. Frost, Maximum autocorrelations for moving average processes, Biometrika 61 (1974), 199-201. MR 51:11889

20. H. Dym and H. P. McKean, Gaussian processes, function theory, and the inverse spectral problem, Academic Press, New York, 1976. MR 56:6829]

21. M. L. Eaton, On the projections of isotropic distributions, Ann. Statist. 9 (1981), 391-400. MR 82c:60026

22. W. Feller, An introduction to probability theory and its applications, vol. II, second ed., Wiley, New York, 1971. MR 42:5292

23. B. R. Frieden, Maximum attainable MTF for rotationally symmetric lenses, J. Opt. Soc. Am. 59 (1969), 402-406.

24. A. Garsia, E. Rodemich, and H. Rumsey, On some extremal positive definite functions, J. Math. Mech. 18 (1969), 805-834. MR 40:4682

25. O. Glatter, The interpretation of real-space information from small-angle scattering experiments, J. Appl. Cryst. 12 (1979), 166-175.

26. Convolution square-root of band-limited symmetrical functions and its application to small-angle scattering data, J. Appl. Cryst. 14 (1981), 101-108.

27. T. Gneiting, On $\alpha$-symmetric multivariate characteristic functions, J. Multivariate Anal. 64 (1998), 131-147. MR 99h:60025

28. —_ Radial positive definite functions generated by Euclid's hat, J. Multivariate Anal. 69 (1999), 88-119. MR 2000g:60022

29. Criteria of Pólya type for radial positive definite functions, Proc. Amer. Math. Soc. 129 (2001), 2309-2318. MR 2002b:42018 
30. - Compactly supported correlation functions, J. Multivariate Anal. 83 (2002), 493-508. MR 2003i: 60057

31. T. Gneiting, K. Konis, and D. Richards, Experimental approaches to Kuttner's problem, Experiment. Math. 10 (2001), 117-124.

32. B. I. Golubov, On Abel-Poisson type and Riesz means, Anal. Math. 7 (1981), 161-184. MR 83b:42015

33. D. V. Gorbachev, Extremum problem for periodic functions supported in a ball, Math. Notes 69 (2001), 313-319. MR 2002e:42006

34. E. A. Gorin, Extremal rays in cones of entire functions, Abstracts, XIII Soviet Workshop on Operator Theory in Function Spaces, Siberian Academy of Sciences, 1988, pp. 57-58.

35. I. S. Gradshteyn and I. M. Ryzhik, Table of integrals, series and products, fifth ed., Academic Press, Boston, 1994. MR 94g:00008

36. E. M. Hofstetter, Construction of time-limited functions with specified autocorrelation functions, IEEE Trans. Info. The. IT-10 (1964), 119-126.

37. M. E. H. Ismail and M. E. Muldoon, On the variation with respect to a parameter of zeros of Bessel and q-Bessel functions, J. Math. Anal. Appl. 135 (1988), 187-207. MR 89i:33011

38. A. J. E. M. Janssen, Frequency-domain bounds for non-negative band-limited functions, Philips J. Res. 45 (1990), 325-366.

39. , Bounds for optical transfer functions: analytical results, Philips J. Res. 45 (1991), $367-411$.

40. M. Kanter, Unimodal spectral windows, Statist. Prob. Lett. 34 (1997), 403-411. MR 98e:60025

41. M. N. Kolountzakis and S. G. Révész, On a problem of Turán about positive definite functions, Proc. Amer. Math. Soc. 131 (2003), 3423-3430.

42. - On pointwise estimates of positive definite functions with given support, preprint (2003); available from http://fourier.math.uoc.gr/ mk/publ/.

43. M. Krein, Sur le probléme du prolongement des fonctions hermitiennes positives et continues, C. R. (Doklady) Acad. Sci. URSS (N. S.) 26 (1940), 17-22. MR 2:361h

44. B. Kuttner, On the Riesz means of a Fourier series (II), J. London Math. Soc. 19 (1944), 77-84. MR 7:59d

45. W. Lawton, Uniqueness results for the phase-retrieval problem for radial functions, J. Opt. Soc. Am. 71 (1981), 1519-1522. MR 82m:94010

46. W. Lukosz, Properties of linear low-pass filters for nonnegative signals, J. Opt. Soc. Am. 52 (1962), 827-829

47. _ Übertragung nichtnegativer Signale durch lineare Filter, Optica Acta 9 (1962), 335364 .

48. A. Mantoglou and J. L. Wilson, The turning bands method for simulation of random fields using line generation by a spectral method, Water Resour. Res. 18 (1982), 1379-1394.

49. R. P. Millane, Phase retrieval in crystallography and optics, J. Opt. Soc. Am. A7 (1990), 394-411.

50. F. Natterer, The mathematics of computerized tomography, Teubner, Stuttgart, 1986. MR 88m:44008

51. D. S. Oliver, Moving averages for Gaussian simulation in two and three dimensions, Math. Geol. 27 (1995), 939-960. MR 96k:86013

52. R. E. A. C. Paley and N. Wiener, Fourier transforms in the complex domain, American Mathematical Society, New York, 1934. MR 98a:01023

53. A. Papoulis, Apodization of optimum imaging of smooth objects, J. Opt. Soc. Am. 62 (1972), $1423-1429$.

54. Minimum-bias windows for high-resolution spectral estimates, IEEE Trans. Info. The. IT-19 (1973), 9-12.

55. M. Plancherel and G. Pólya, Fonctions entières et intégrales de Fourier multiples, Comment. Math. Helv. 9 (1937), 224-248.

56. J. Rosenblatt, Phase retrieval, Commun. Math. Phys. 95 (1984), 317-343. MR 86k:82075

57. W. Rudin, An extension theorem for positive-definite functions, Duke Math. J. 95 (1970), 49-53. MR 40:7722

58. I. J. Schoenberg, Metric spaces and completely monotone functions, Ann. of Math. 39 (1938), 811-841. 
59. H. Stark and B. Dimitriadis, Minimum-bias spectral estimation with a coherent optical spectrum analyzer, J. Opt. Soc. Am. 65 (1975), 425-431, Errata 65 (1975), 973.

60. A. Walther, The question of phase retrieval in optics, Optica Acta 10 (1963), 41-49. MR 29:890

61. C. S. Williams and O. A. Becklund, Introduction to the optical transfer function, Wiley, New York, 1989.

62. V. P. Zastavnyi, On positive definiteness of some functions, J. Multivariate Anal. 73 (2000), 53-81. MR 2002b:42017

63. - Positive definite radial functions and splines, Dokl. Math. 66 (2002), 446-449.

64. V. P. Zastavnyi and R. M. Trigub, Positive definite splines of special form, Mat. Sb. 193 (2002), 41-68.

Institut für Grenzgebiete der Psychologie und Psychohygiene, Wilhelmstrasse 3A, 79098 Freiburg, Germany

E-mail address: ehm@igpp.de

Department of Statistics, University of Washington, Box 354322, Seattle, WashingTON 98195-4322

E-mail address: tilmann@stat.washington.edu

Department of Statistics, Pennsylvania State University, 326 Thomas Bullding, UniVersity Park, Pennsylvania 16802-2111

E-mail address: richards@stat.psu.edu 